\title{
Room-Wide Wireless Charging and Load-Modulation Communication via Quasistatic Cavity Resonance
}

\author{
TAKUYA SASATANI, The University of Tokyo, Disney Research, and JSPS Research Fellow \\ CHOUCHANG (JACK) YANG, Disney Research \\ MATTHEW J. CHABALKO, Disney Research \\ YOSHIHIRO KAWAHARA, The University of Tokyo \\ ALANSON P. SAMPLE, University of Michigan
}

\begin{abstract}
The rise of the Internet of Things (IoT) has led to a significant increase in the number of connected devices that stream data in our homes, offices and industrial spaces. However, as the number of these devices increases, the costs of actively maintaining and replacing batteries becomes prohibitive at scale. Recent work on Quasistatic Cavity Resonance (QSCR), offers the possibility of seamless wireless power transfer (WPT) to receivers placed anywhere inside large indoor spaces. This work aims to solve two unexplored and critical missing pieces needed to realize this vision of ubiquitous WPT. First, we demonstrate a full end-to-end QSCR-based WPT system that is capable of simultaneously charging multiple custom designed nodes nearly anywhere in the $4.9 \mathrm{~m} \times 4.9 \mathrm{~m} \times 2.3 \mathrm{~m}$ test room. Second, this work utilizes the WPT mechanism as a communication channel, where nodes communicate with a centralized reader and to each other via load modulation. Through analysis and experiments, the proposed system shows that 10 receiver nodes can be safely and efficiently wirelessly charged and the end node to end node communication rate can achieve from $1 \mathrm{kbps}$ without occurring any errors, up to $5 \mathrm{kbps}$ with $6 \%$ BER while the end node to central unit can achieve $10 \mathrm{kbps}$ without occurring any errors.
\end{abstract}

CCS Concepts: • Human-centered computing $\rightarrow$ Ubiquitous and mobile computing; $\bullet$ Hardware $\rightarrow$ Communication hardware, interfaces and storage;

Additional Key Words and Phrases: Wireless sensor networks, wireless power transfer, load-modulation communication, quasistatic cavity resonance

ACM Reference Format:

Takuya Sasatani, Chouchang (Jack) Yang, Matthew J. Chabalko, Yoshihiro Kawahara, and Alanson P. Sample. 2018. Room-Wide Wireless Charging and Load-Modulation Communication via Quasistatic Cavity Resonance. Proc. ACM Interact. Mob. Wearable Ubiquitous Technol. 2, 4, Article 188 (December 2018), 23 pages. https://doi.org/10.1145/3287066

\section{INTRODUCTION}

Internet of Things (IoT), which connect numerous ambient devices to cooperate with each other is a promising basis for the era of smart environments [1, 23, 28, 30]. The potential impact of IoT is not merely guaranteed to enhancing convenience in everyday-life; it has the potential to overturn the structure of industry, economy, health-care, and so on, by linking various physical phenomenon through the digital domain [1]. As IoT systems

Authors' addresses: Takuya Sasatani, The University of Tokyo, Disney Research, JSPS Research Fellow, sasatani@akg.t.u-tokyo.ac.jp; Chouchang (Jack) Yang, Disney Research, jack.yang@disneyresearch.com; Matthew J. Chabalko, Disney Research, matt.chabalko@ disneyresearch.com; Yoshihiro Kawahara, The University of Tokyo; Alanson P. Sample, University of Michigan, apsample@umich.edu.

Permission to make digital or hard copies of all or part of this work for personal or classroom use is granted without fee provided that copies are not made or distributed for profit or commercial advantage and that copies bear this notice and the full citation on the first page. Copyrights for components of this work owned by others than the author(s) must be honored. Abstracting with credit is permitted. To copy otherwise, or republish, to post on servers or to redistribute to lists, requires prior specific permission and/or a fee. Request permissions from permissions@acm.org.

(C) 2018 Copyright held by the owner/author(s). Publication rights licensed to ACM.

2474-9567/2018/12-ART188 \$15.00

https://doi.org/10.1145/3287066

Proc. ACM Interact. Mob. Wearable Ubiquitous Technol., Vol. 2, No. 4, Article 188. Publication date: December 2018. 
are rapidly gaining ground, the number of computation "nodes" which act as interfaces, sensors, and actuators are increasing $[1,23]$. This trend is boosting currently; the academic, industrial, and hobby communities are on the way of building a whole new class of applications by invisibly weaving computation in our everyday-lives [45].

Nowadays, studies that develop these IoT devices make prototype systems with a small number of devices; these are typically powered by batteries or wires and communicate using power-hungry radio transmitters. However, envisioning the future that hundreds of everyday-objects contained in a 3-D space become computation nodes, it is obvious that the cost of battery charging/replacement and communication becomes prohibitive at scale $[2,47]$. One emerging counterpart is to make devices work in ultra-low-power levels (e.g., micro-Watt order) and powering them via microwave power transfer or RF energy harvesting [24, 34]. Although this direction may be powerful for driving minimum primitive functions in sparse, outdoor environments (i.e., environments where line-of-sight can easily be achieved), the available functions are strictly limited due to the lack of energy and many fundamental challenges exist for operating in typical indoor environments (i.e., environments where external interference and out-of-sight conditions easily occur). Given that in modern life, a considerable amount of activities take place in indoor environments (e.g., work in office spaces, leisure at homes, manufacturing in factories, health-care at hospitals, etc), this missing piece for IoT is critical. We aim to construct sustainable IoT platforms in indoor environments by enabling the following two key concepts: ubiquitous wireless power transfer (WPT) and low-power communication.

As for WPT, the mainstream of system architecture is a simple "central-to-node" form, whereas there are many "physical" challenges to achieve ubiquitous WPT at power levels higher than several Watts (e.g., safety, efficiency, lack of range, etc). On the other hand, communication has a rather complex architecture; up-link/down-link, node-to-node, broadcasting, and other directions exist in practice. Although, it is known that as long as one-to-one communication in the "physical layer" can be established between every pair of devices, everything else necessary to work as a whole has a reasonable solution in the MAC layer $[8,46]$. The problem here is that it is difficult to establish robust communication links with low-power devices. Low-power RF communication devices (e.g., UHF RFID tags) usually suffer in out-of-sight conditions as well as placements alongside exile dielectric/conductive media, which both occasions are not rare for indoor mobile objects. From these observations, we believe that the most urgent bottlenecks of IoT left to be solved lies in the physical layer of power supply and communication; this study aims to provide a solution for this urgent piece.

Various approaches tackled the above-mentioned bottlenecks lying in the physical layer of IoT. However, none of them succeeded to simultaneously fulfill the following requirements; our belief is that these are necessary for practical indoor IoT systems:

\section{Requirement 1}

Safe wireless power transfer in power levels over several Watts. The future we should aim for is to safely and autonomously power hundreds of randomly placed nodes with various (including rich) functions. This requires magnitudes of higher total power levels compared to prior works on ubiquitous WPT; therefore we believe that it is an important direction to establish safe and ubiquitous WPT with high power levels.

\section{Requirement 2}

Robust operation everywhere in a 3-D volume, including out-of-sight configurations. Typically, any object with mobility can be easily placed in random positions, or even out-of-sight by being placed in drawers, stuffed in pockets, shaded by other objects, etc. Therefore, it is important that the nodes can operate in a wide range of positions, including out-of-sight configurations. Our belief is that enhancing the range and robustness of WPT/communication is more urgent than increasing data rate since primitive functions of IoT, such as sensing and actuation don't usually require high-data rates.

\section{Requirement 3}

Inherently cheap node structures. Assuming that every ambient object becomes IoT nodes, it is vital that the components used for power reception and communication can be manufactured cheaply in mass-production. 

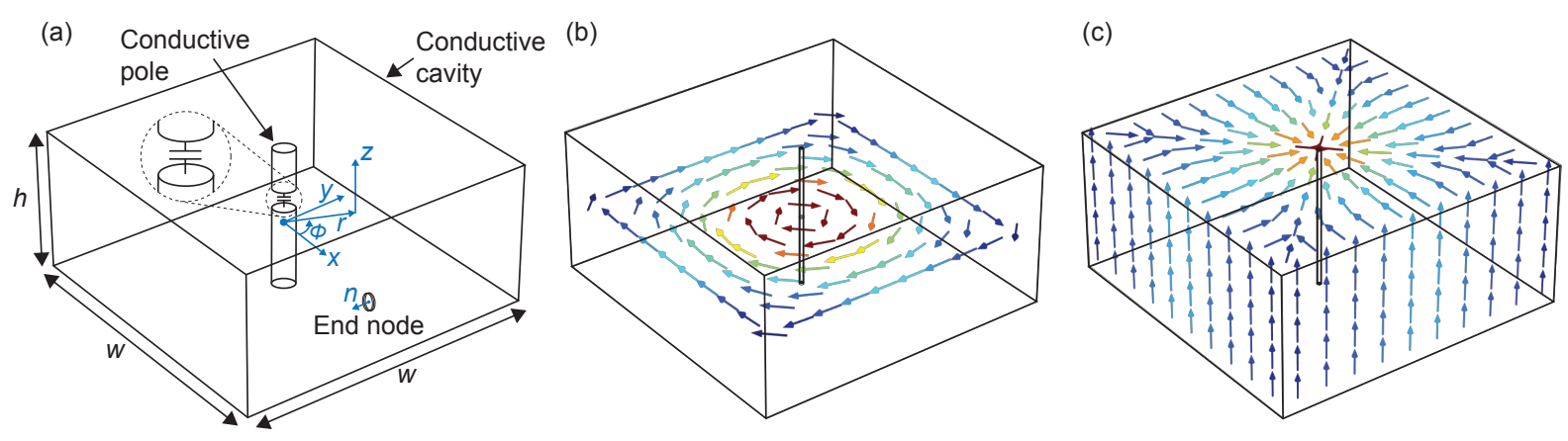

Fig. 1. Overview of QSCR. When the resonant mode is excited, the current flows through the "pole-ceiling-wall-floor-pole" loop, whereas the magnetic flux swirls around the central conductive pole. (a) The structure of QSCR. An end node is depicted as well as a coil resonator. (b) The magnetic field generated by the QSCR. (c) The Surface current generated by the QSCR. Color is magnitude (red, large; blue, small).

Our belief is that minimizing cost in the prototyping stage is not vital, although designing nodes so they can be easily exported to low-cost manufacturing is a solid requirement.

Here, we note that the term of safety in WPT is multifold, although the most fundamental measure adopted by ICNIRP, IEEE, and FCC guidelines is specific absorption rate (SAR) [6]. We follow this measure, although struggling with the regulation thresholds is not the essential point; our definition of safe WPT systems are systems that can keep the "SAR per delivered power" low, considering the linear nature of typical WPT systems.

Ubiquitous WPT, which seamlessly and safely powers numerous receiver nodes, randomly placed in a large 3-D volume, has been an unsolved challenge for long [3, 12]. WPT via magnetic resonant coupling suffered from the limitation of power delivery distance, whereas microwave-based methods have fundamental safety challenges. Recently, a new route towards ubiquitous WPT was proposed using quasistatic cavity resonators (QSCR), which can power numerous devices contained in a large 3-D volume using magnetic induction $[5,35,36]$. QSCR is a metallic cavity containing a central conductive pole with discrete capacitors inserted in a gap in the pole, such that one of the resonant modes of the cavity enters quasistatic operation [See figure 1]. This allows magnetic fields to permeate the interior of the cavity while confining the cumbersome electric fields to the discrete capacitors.

This QSCR technology has three vital benefits for ubiquitous WPT in indoor environments, which were not available collectively in former techniques: (i) the capability to transmit power to small footprint receivers, (ii) a room-scale power delivery range including out-of-sight areas, and (iii) the minimum interference with external objects which lead to safe operation. Even in the case of using receivers with footprints small as 1/1000 of the transmitter, high efficiency (over 50\%) power transfer was demonstrated throughout most of the volume in a room-scale $(4.9 \mathrm{~m} \times 4.9 \mathrm{~m} \times 2.3 \mathrm{~m})$ QSCR. Moreover, QSCR-based systems are hardly affected by people or furniture shading the receiver or existing in the cavity volume. This is because magnetic fields in around a few $\mathrm{MHz}$ doesn't interfere much with human bodies or everyday objects when compared to electric fields; consequently, safe WPT at high power levels can be achieved, even in out-of-sight configurations [5, 6]. These characteristics make the QSCR technology one of the most promising candidate for achieving ubiquitous WPT in indoor environments.

The robust nature of the QSCR channel (i.e., magnetic field) was only explored for ubiquitous WPT means. However, power transfer and communication are inherently similar in terms of physics; by reusing the QSCR channel also for data transfer should light the way towards achieving robust communication everywhere including out-of-sight placements. This is one of the above-mentioned requirements which RF backscatter-based systems 
suffer from. Here we come to notice that although the primitives of QSCR as a power transfer link has been studied in former studies, the two following critical pieces have not been explored yet: (i) constructing a full end-to-end WPT system that is capable of simultaneously charging multiple custom designed nodes, and (ii) analysis, implementation, evaluation, and demonstration of QSCR as a load-modulation based data transfer link $[5,35]$. This brings about two challenges that we need to overcome: handling the extremely large dynamic range of the incoming signals and providing a practical guideline for node design.

Since our system: (i) operates in near-field, (ii) the coupling between the transmitter and the receiver intensely changes, and (iii) power/data transfer links co-exist, the dynamic range of the incoming signals become extremely large in naively designed systems. This is a rather minor challenge in far-field systems and conventional near-field communication (NFC) systems, since far-field systems have nearly static antenna impedance, whereas NFC doesn't assume large variations in receiver positions; this is a challenge specific to our system which needs to be solved for. We overcome these challenges by implementing a hardware/software-combined decoding circuit; this circuit dynamically adapts the incoming signal levels to a fixed range through software control, whereas the hardware partition protects the component from fast voltage fluctuations.

As for the practical guideline of node design, conventional coil-based systems have many convenient methods to extract circuit parameters from the geometrical property (e.g., Neumann formula, magnetic dipole approximation, etc); however, the current flowing in the QSCR distributes within a conductive plane, not focused in a conductive wire; this property inhibits the direct application of traditional analysis methods. Therefore, we provide a simplified formula to extract the coupling coefficients from the geometrical properties (e.g. cavity size, receiver size, turns of coils, etc) based on former studies on QSCR [5]. We also evaluate the communication and power transfer capabilities depending on the value of coupling coefficient; given that in near-field systems, interference between two coupled circuits can be fully characterized by coupling coefficient, this provides a practical guideline for future system design.

Our prototype system consists of a room-scale $(4.9 \mathrm{~m} \times 4.9 \mathrm{~m} \times 2.3 \mathrm{~m}) \mathrm{QSCR}$, ten custom designed node units, and a central unit; power is delivered to the nodes using the resonant frequency of the QSCR: $1.325 \mathrm{MHz}$, and communicates in bi-direction using a carrier frequency of $1.325 \mathrm{MHz}$ and a modulation frequency of 1-to-50 kHz. Our custom designed nodes are capable of receiving power from the QSCR as well as bi-direction communication via load-modulation; moreover, they are composed by low-power, low-cost components (e.g., opamps, micro-controllers, MOSFETs, etc).

In summary, the contributions of this study are as follows:

\section{Contribution 1}

Demonstration of a full end-to-end QSCR-based WPT system, which can simultaneously charge multiple custom designed receiver nodes nearly anywhere in the $4.9 \mathrm{~m} \times 4.9 \mathrm{~m} \times 2.3 \mathrm{~m}$ test room. Our receiver nodes are equipped with a microcontroller, a communication front-end, a battery, and a power management unit, which together work as primitives of IoT devices, despite previous work empowered multiple LEDs [5]; the difference in power level and the non-static load profiles (i.e. typical devices, including our node vary its input impedance depending on the operation state) makes this advancement non-obvious. We emulated a typical indoor scenario by randomly placing 10 receiver nodes inside a furnished room and showed that these nodes can be simultaneously charged wirelessly.

\section{Contribution 2}

A theoretical analysis and simplified formulation of the QSCR channel, followed by evaluations of communication and power transfer when the coupling is varied; this works as a practical guideline for the design of future QSCR-based systems, which cannot be analyzed by conventional analysis methods.

\section{Contribution 3}

Design, implementation, and evaluations of a QSCR-based load-modulation communication system integrated with WPT functions. In order to handle the extreme dynamic range of incoming signals without 
distortions or voltage breakdown, we custom design a low-cost hardware/software-combined decoding circuit. As the first study to attempt load-modulation communication on the QSCR channel, our focus is to explore the basic characteristics of the channel and provide an experimental proof of concept. Therefore, we use binary ASK (i.e., OOK: ON-OFF-Keying) as this is one of the most simple modulation techniques.

First, in this paper, a brief tutorial about related works are presented to motivate our work. Then, the overview of the studied power/data transfer system is provided and the QSCR as both power and data transfer links are investigated through circuit theory-based analysis. Next, a QSCR-based power/data transfer system is designed and implemented. Finally, the performance of the system from both power/data transfer aspects is evaluated through numerical analysis and measurements, which is followed by discussions and conclusions.

\section{RELATED WORKS}

Ubiquitous WPT and low-power communication are well known big challenges for long; there were many attempts to enable these concepts in the real world $[2,34]$. Major methods for ubiquitous WPT include microwave, optical (i.e. laser), magnetic resonant coupling, cavity resonator, and the recently presented quasistatic cavity resonance (QSCR) [39]. On the other hand, the majority of low-power communication projects are driven by semi-passive (e.g., load-modulation, backscatter) technologies; our study also follows this growing trend. This section gives an overview of these methods, examine if they are attainable for our three above-mentioned requirements, and motivates our solution using QSCR-based WPT and load-modulation communication.

\subsection{Microwave}

Known as one of the methods with the longest potential power delivery ranges, microwave power transfer (MPT) is a method that transfers power via far-field electromagnetic waves (typically around a $\mathrm{GHz}$ ) [2, 3, 38]. A transmitter antenna radiates energy and the receiver antenna absorbs this radiated energy; this is the same principle used in many wireless communication links (e.g., Wi-Fi, Cellular, Bluetooth, etc). This similarity inspired another class of ubiquitous power sources: RF energy harvesting, where the nodes acquire energy from the ambient electromagnetic waves used for wireless communication [14, 18, 20, 24, 25]. RF energy harvesting is gaining attention since it shows the possibility to generate a few microwatts of power out of thin air, considering that TV, cellular, and Wi-Fi networks exist everywhere in contemporary urban environments.

Although there are specific applications that can operate in a few microwatts, this is still a very strict energy constraint, which strongly limits the variety of available applications. Considered together with the low system efficiency of MPT, this brings about the necessity for a dedicated power source with magnitudes of higher power levels (more than a few watts); however, this leads to two vital issues. The first issue is that the specific absorption rate (SAR) of microwaves around GHz are typically high, which means that such high-power microwaves can easily heat up human bodies and cause health problems [50]. The second issue is the distortion of communication links [13]. It is known that even in nearby frequency bands, the communication quality gets distorted by high-power microwaves; due to the drought of vacant frequency bands and strict regulations, it is not a realistic plan to ensure a dedicated frequency band far away from existing communication bands, therefore this distortion is a critical issue.

Other challenges of MPT which should be noted are the restriction of antenna size and limitations in out-of-sight links. When good performance (e.g. high-gain, high-efficiency, etc) is desired, the antenna size has to be around half-wavelength $(\sim 150 \mathrm{~mm}$ for $1 \mathrm{GHz})$ and the performance typically degrades as the antenna size gets smaller. As for the out-of-sight links, many projects and start-ups are attempting to apply retro-directive systems, which enables focusing electromagnetic energy to an intended point [26, 49]. However, constructive interference at non-desired points can easily occur, which will question the safety of the system. From these considerations, our 
opinion is that although MPT is useful for specific ultra-low-power applications, it still has many fundamental challenges towards achieving our goal of robust and safe power transfer in power levels over several Watts.

\subsection{Optical (Laser)}

Optical approaches, which transfers energy via laser beams is another method that is gaining attention [3, 13]. Laser beams are a subset of electromagnetic waves in which the wavelength are magnitudes smaller $(\sim$ a micrometer) than microwaves ( around a few hundred millimeters). This ultra-small wavelength enables highly directive radiation; while this can favor the extension of operation range and miniaturization of receivers, it also substantially guarantees the conditions of safe operation into line-of-sight configurations.

The laser beams are generated by laser diodes, which converts current into laser beams (i.e., carrier re-generation effect) and absorbed by photovoltaic cells, which convert light into current (i.e., carrier generation effect). The critical problem that lies here is the low conversion efficiency of photovoltaic cells ( $\sim 20 \%$ [13]), which severely degrades system efficiency. Moreover, $20 \%$ conversion efficiency means that 4 times the power supplied to the device is directly converted to "heat" at the device, which causes inevitable thermal issues. Recent studies attempted to solve this problem by developing custom designed, small form-factor heat-sinks [13]. While these worked at some extent in lab environments, there need to be many more evaluations in practical situations, such as the performance when it is in contact with small thermal convection media.

Also, due to the short wavelength of light, the energy easily gets absorbed in biological tissues and damage eyes, which both lead to severe health problems. The use of a fundamentally hazardous channel makes it difficult to make a fail-safe architecture. A recent study attempts to detour this problem by "stopping" power transfer when exile objects come across; this way, the power supply is guaranteed to line-of-sight conditions with no users or ambient objects nearby [13].

\subsection{Magnetic Resonant Coupling}

Introduced by MIT in 2007, magnetic resonant coupling-based WPT is a method that transfers energy via the inductive link between the transmitting and receiving resonators. This method offers the promise for high-efficiency and safe power delivery in mid-range distances (i.e., approximately, the size of the resonators) [6, $21,43]$. From the circuit theory point of view, magnetic resonant coupling is an extension of inductive WPT; inductive WPT is a method that enables high-efficiency power delivery between short distances and is used in Qi standards $[19,31,44]$. The difference between the two methods is that inductive WPT uses a pair of ordinary coils, whereas magnetic resonant coupling uses a pair of high- $Q L C$ resonators; this high- $Q$ resonance successfully enhances the power delivery range.

Albeit the high efficiency of magnetic resonant coupling-based WPT, the limited power delivery range and the degradation of efficiency in asymmetrical (i.e., receiver is much smaller than the transmitter) systems are still vital limitations for use in ubiquitous WPT. Some studies attempt to extend the power delivery range by using 2-D resonator arrays as transmitters [37]; this makes a 2-D area of power delivery range, although the extension of this range to a 3-D volume remains as a big challenge. Near-field 2-D phased array techniques, which aims to enhance power delivery range by making a constructive interference at the receiver are also considered [15, 22]; although these methods show a strong advantage when the angle of the receiver is misaligned, it doesn't show a significant difference in long-distance configurations, which is necessary for 3-D WPT using 2-D arrays [17].

\subsection{Cavity Resonators}

Cavity resonators, which are the ancestor of the later explained QSCR are well known for the ability to generate 3-D near-field electromagnetic field patterns (i.e., resonant modes) in a metallic cavity. There were many attempts to directly apply cavity resonators for 3-D WPT since they naturally withhold a 3-D power supply range, 


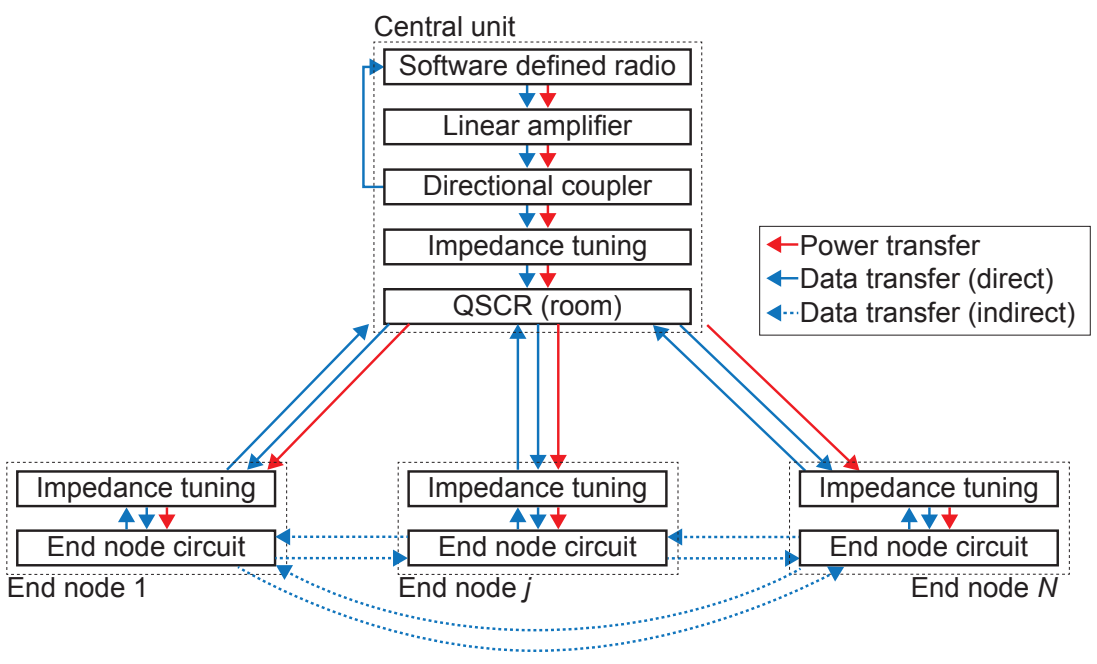

Fig. 2. The functional diagram of the implemented power/data transfer system using QSCR and load-modulation. The data transfer links between multiple end nodes are considered as "indirect" links since they talk through the magnetic channel generated by the QSCR.

although they suffered from two vital challenges: the co-existence of intense electrical and magnetic field, and the resonant frequency uniquely fixed by the cavity dimensions [4,27]. Electric fields are easily distorted by dielectrics (e.g., human body, water, pieces of wood, etc), while magnetic fields are less affected by everyday objects. Therefore, our belief is that for ubiquitous WPT, it is ideal to suppress the electrical field and make the magnetic field dominant in the channel (i.e., magneto-quasistatic operation). Also, the lack of control in resonant frequency means that the system has to operate in different frequencies in different sized rooms; while this limitation may be acceptable in specific industrial applications, it is a barrier for adoption to practical systems from both technical and regulatory aspects.

\subsection{Quasistatic Cavity Resonators (QSCR)}

Quasistatic cavity resonators (QSCR) are a recently developed technology that has the promise of ubiquitous power delivery in large 3-D volumes [5, 35, 36]. QSCR is a technique that compensates for the disadvantages of cavity resonators (i.e., interference with dielectric, resonant frequency fixed by geometry, etc) while preserving the 3-D nature. This technique uses enclosed metallic cavities (i.e., cavity resonators) containing a central conductive pole with discrete capacitors inserted in a gap in the pole, such that one of the resonant modes of the cavity enters deep sub-wavelength (i.e., quasistatic) operation [See Figure 1]. This allows the magnetic fields to permeate the interior of the cavity while confining electric fields to the discrete capacitors; as we mentioned in the cavity resonator section, this is very close to what we think ideal for ubiquitous WPT. Moreover, since the cavity operates in deep sub-wavelength domains, the resonant frequency is structure independent and tunable. The operating principles of QSCR are similar to magnetic resonance coupling when it is abstracted to circuit analysis level [5]. Through simulations and measurements, high efficiency $(>50 \%)$ power transfer to receivers with a footprint of around $1 / 1000$ of the cavity was demonstrated throughout most of the volume within a room-sized $(4.9 \mathrm{~m} \times 4.9 \mathrm{~m} \times 2.3 \mathrm{~m})$ QSCR [5]. Moreover, simulations showed that QSCR can deliver hundreds of Watts of power to mobile devices within regulation limits [5]; transferring this level of power is not our intention, but 


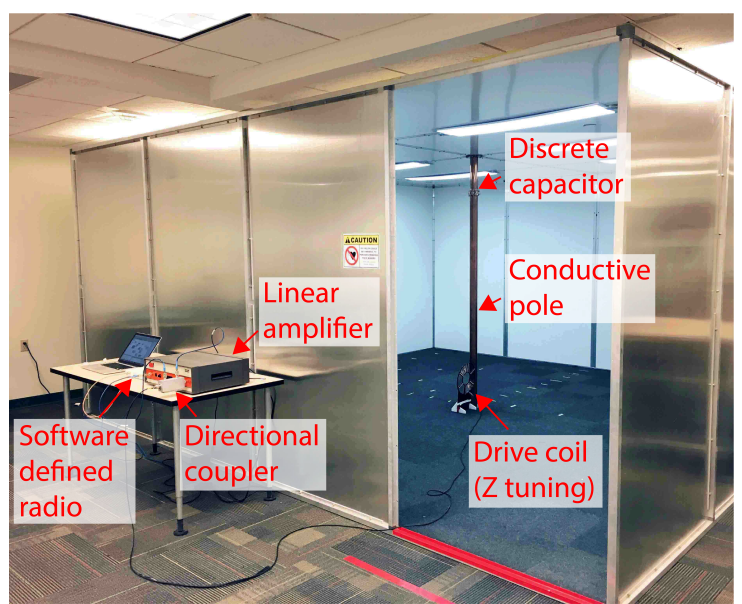

(a)

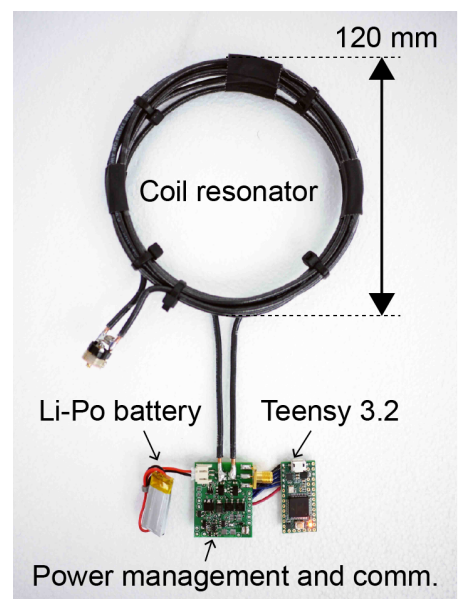

(b)

Fig. 3. (a) Experimental setup of the QSCR-based power/data transfer system. The input impedance of the system can be adjusted by varying the position of the drive coil, which works as a T-matching network. (b) Implemented end node.

our argument is that this nature of QSCR provides a sufficiently large margin under the safety thresholds for delivering more power than a several Watts.

\subsection{Backscatter/Load-Modulation}

Backscatter and load-modulation are techniques that enable nodes to communicate in a "semi-passive" manner; they are in practical use as passive RFIDs and NFC tags [7, 9, 24, 40, 41, 44, 47]. Compared to active radio transmitters (i.e. bluetooth, Wi-Fi, Zigbee, etc), where local oscillators are employed to generate the carrier, it is known that backscatter and load-modulation techniques have the promise for magnitudes of lower energy consumption; it should also be remarked that these schemes reuse the spectrum when configured on the WPT frequency band. We consider these two factors important for future IoT systems since the power consumption is vital and the general depletion of the spectrum.

In a backscatter/load-modulation-based communication system, an external source generates the carrier and the data transmitter (i.e., communication nodes) modulates this carrier by changing the phase/amplitude of the reflection; the term backscatter is used for far-field systems and load-modulation is used for near-field systems. From another viewpoint, this process may be described that the external source is "off-loading" the energy-hungry, carrier generation routine from the nodes; this allows each node to communicate with minimum energy consumption, which expands the lifetime of nodes. This is important since in many cases, end nodes have tight battery capacity limitations.

\section{QSCR-BASED POWER/DATA TRANSFER SYSTEM}

\subsection{System Overview}

Functional architecture. The simplified functional diagram of the studied QSCR-based WPT and load-modulation communication system is shown in Figure 2 and the corresponding experimental setup is shown in Figure 3. The current and magnetic field distributions of the QSCR in resonance are shown in Figure 1 


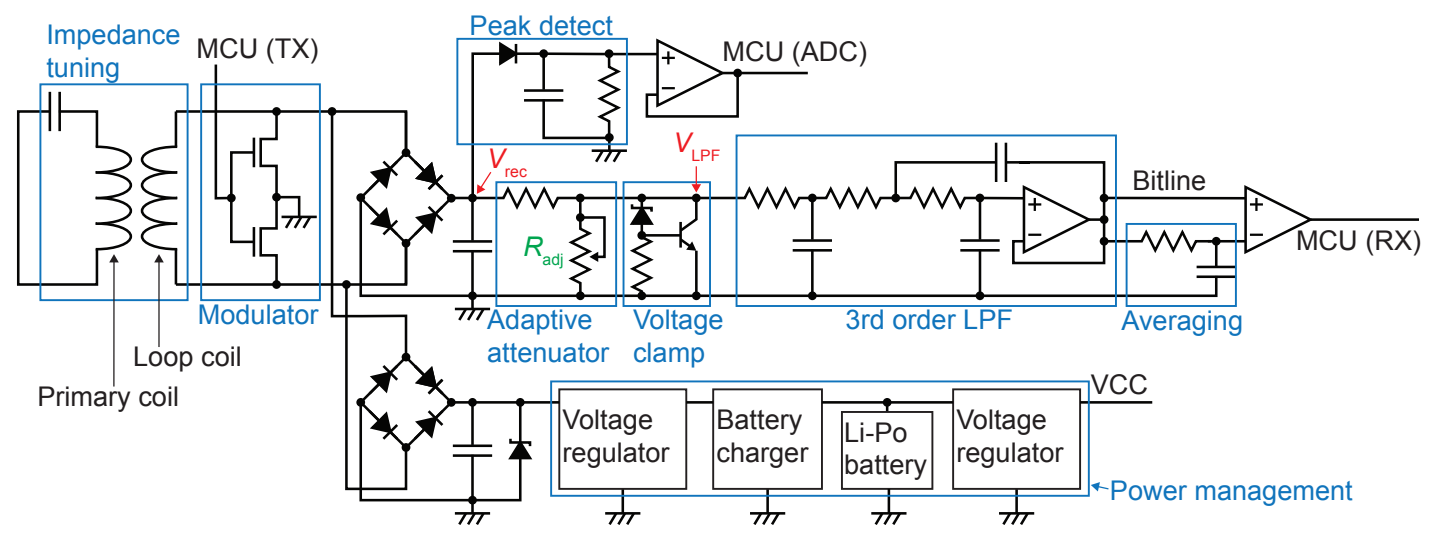

Fig. 4. Simplified circuit diagram of the end nodes. TX and RX stand for transmitter and receiver, respectively.

as well for reference; the current flows through the "pole-ceiling-wall-floor-pole" loop, whereas the magnetic flux swirls around the central conductive pole. Looking at the big picture, the system consists of one "central unit" and multiple "end nodes". The central unit consists of an Ettus X310 software-defined radio (SDR), a $50 \Omega$ linear amplifier, a directional coupler, and the QSCR (room) [See Figure 2]. The end nodes consist of a coil resonator, a loop coil for impedance tuning, an encode/decode circuit for load modulation, a Li-Po battery, and a power management unit [See Figure 4]. The coil resonator here refers to a series $L C$ resonator; this resonator consists of a coil which inductively couples to the ambient magnetic field generated by the QSCR and a series capacitor which confines the electrical energy and fixes the resonant frequency.

Design of resonators. The QSCR was fabricated as shown in Figure 1, with the dimensions of $4.9 \mathrm{~m} \times 4.9 \mathrm{~m} \times 2.3 \mathrm{~m}$. The floor, wall, and ceiling are made of aluminum sheet metal bolted on to an aluminum frame, whereas the central conductive pole is made of a copper pole with a diameter of $7.2 \mathrm{~cm} ; 15 \mathrm{high}-\mathrm{Q}$ discrete capacitors are inserted across a $2.5 \mathrm{~cm}$ gap in the pole to fix the resonant frequency. A $1.2 \mathrm{~m} \times 2.3 \mathrm{~m}$ opening serves as a door; it is shown in former studies that this missing panel had a negligible effect on system performance [5]. As shown in Figure 3a, a $28 \mathrm{~cm}$ diameter, 8-turn, spiral drive coil is used to stimulate the resonant mode of the QSCR; this drive coil also works as an adjustable impedance tuning network, therefore the input impedance of the system can be adjusted by the position of this drive coil. The receiver coil is composed of a 5 turn coil with a diameter of $120 \mathrm{~mm}$, fabricated by AWG 12 copper wire; the inductance of this coil was $5.1 \mu \mathrm{H}$. The resonant frequency $f_{0}$ of the QSCR and the coil resonators were tuned to $1.325 \mathrm{MHz}$ under the condition shown in Equation 1 [5] (i.e., series resonance).

$$
\omega_{0}=2 \pi f_{0}=\frac{1}{\sqrt{L C}}
$$

Here, the angular resonant frequency, the inductance, and the capacitance are represented as $\omega_{0}, L$, and $C$, respectively. Using standard RF measurement techniques [16], the $Q$-factor of the QSCR is found to be 1670 and the $Q$-factor of the receiver coils are 220 .

Central unit to end node power/data transfer. The X310 SDR in here uses a binary ASK signal with a carrier frequency of $1.325 \mathrm{MHz}$ to transmit power and data to the end nodes. It is noted that when the central unit is only transmitting power without transmitting data, the SDR outputs a pure sine wave of $1.325 \mathrm{MHz}$. The amplified signal is input to the QSCR through an impedance tuning network, which generates an ASK modulated magnetic field that permeates the interior of the QSCR. The generated magnetic field interlinks with the coil 


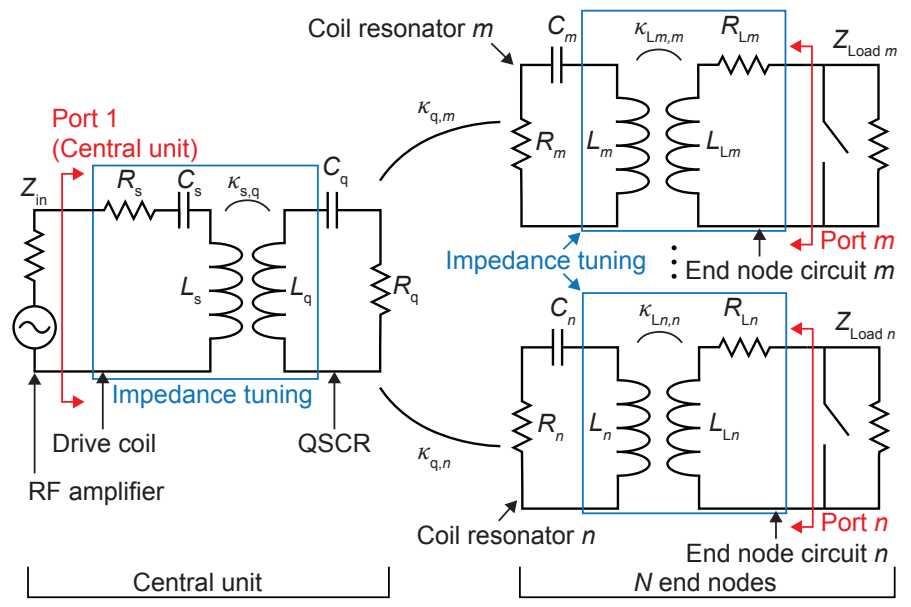

(a)

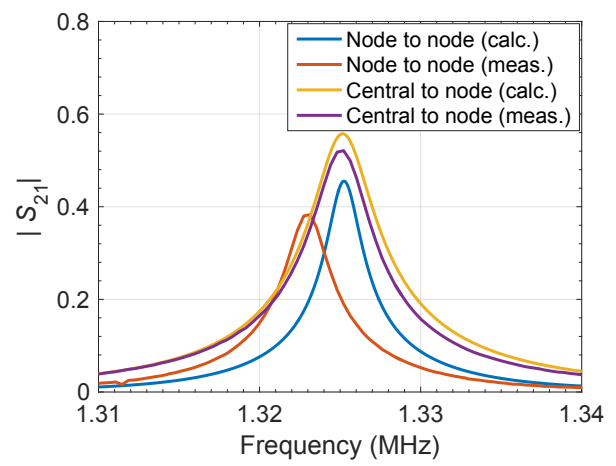

(b)

Fig. 5. (a) Equivalent circuit model of the QSCR-based power/data transfer system. T-matching networks which consists of a coil resonator and a loop coil are used for impedance tuning [32]. The switches on the end nodes are used for load modulation communication. (b) Frequency and the absolute value of the forward voltage gain $\left(\left|S_{21}\right|\right)$. "End node to end node" and "central unit to end node" configurations are considered here. The calculated results were obtained by Equation 13 and 14; the measurement results were obtained by 2-port measurements using a vector network analyzer. The drive coil and coil resonators were placed at $0.45 \mathrm{~m}$ and $1.2 \mathrm{~m}$ from the center of the QSCR, respectively, on a different side of the QSCR. The drive coil position refers to the impedance tuning schema described in Section 5 and the coil resonator position is halfway between the central pole and the wall of the QSCR.

Table 1. Circuit parameters used in Figure 5a

\begin{tabular}{c|c|c|c|c|c|c|c|c|c|c|c|c}
$Z_{\text {in }}, Z_{\mathrm{Load}, i}$ & $L_{\mathrm{s}}$ & $C_{\mathrm{s}}$ & $R_{\mathrm{s}}$ & $L_{\mathrm{q}}$ & $C_{\mathrm{q}}$ & $R_{\mathrm{q}}$ & $L_{i}$ & $C_{i}$ & $R_{i}$ & $L_{\mathrm{L} i}$ & $R_{\mathrm{L} i}$ & $\kappa_{\mathrm{L} i, i}$ \\
\hline $50 \Omega$ & $16 \mu \mathrm{H}$ & $850 \mathrm{pF}$ & $221 \mathrm{~m} \Omega$ & $2.0 \mu \mathrm{H}$ & $7.1 \mathrm{nF}$ & $10 \mathrm{~m} \Omega$ & $5.1 \mu \mathrm{H}$ & $2.8 \mathrm{nF}$ & $190 \mathrm{~m} \Omega$ & $0.34 \mu \mathrm{H}$ & $40 \mathrm{~m} \Omega$ & $3.1 \times 10^{4}$
\end{tabular}

resonators of the custom designed end nodes placed inside the QSCR, which allows the end nodes to receive energy and read out the bits embedded in the magnetic field. This enables both power and data transfer, in the direction from the central unit to the end nodes.

End node to central unit data transfer. The next question should be how the end nodes transmit data to the central unit. The end nodes can modulate the magnetic field by varying the impedance connected to the coil resonators; a MOSFET bridge controlled by the baseband signal is connected to the coil resonators to switch between load and short conditions. The load impedance of the end node affects the input impedance seen from the central unit; consequently, the input current and the intensity of the magnetic field generated in the QSCR is modulated by the baseband signal. This baseband embedded in the magnetic field can be read out at the central unit through the SDR, by measuring the reflected/forward power extracted by the directional coupler. This procedure enables data transfer in the direction from the end node to the central unit.

End node to end node data transfer. Now that the end node can transmit and receive data via the QSCR channel, there is no reason that end node to end node communication cannot be established. By following the same procedure mentioned above, communication between multiple end nodes is enabled. 


\subsection{Analysis of the QSCR-Based Power/Data Transfer Link}

This section provides an analysis of the QSCR channel; the full equations are shown in Appendix A and the effect of external objects present in the channel is discussed in Appendix B. First, in this section, a simplified circuit model of the QSCR is extracted in a closed form. The extracted model is applied for the analysis of the power/data transfer links and is compared with measurement results to validate our analysis. In addition, a brief analysis of wireless power transfer performance is also provided at the end of this section.

Extraction of the circuit model. Since all resonators are in sub-wavelength operation, the couplings between the QSCR and the end nodes are mainly inductive. Therefore, the equivalent circuit of the system can be depicted as Figure 5a. It is noted that the coupling coefficient used here is $\kappa$, which is a definition commonly used in coupled mode theory-based studies and also used in previous QSCR studies $[5,11]$. The transformation from $\kappa$ to the magnetic coupling coefficient $k$, which is frequently used in circuit analysis-based studies can be given by $k=\frac{2 \kappa}{\omega_{0}}$, where $\omega_{0}$ is the angular resonant frequency of the coupled resonators. The couplings between the end nodes are neglected since the QSCR is significantly larger than the end nodes; it can be assumed that these couplings only have a subtle effect on the system unless multiple end nodes are placed just next to each other.

Considering a typical power/data transfer system using coupled resonators, the only parameters in Figure 5a that change over time is the end node positions; the inductance, capacitance, copper-loss, and matching circuit parameters are not likely to change over time. Under this assumption, all parameters except the coupling coefficient between the QSCR and the coil resonators: $\kappa_{\mathrm{q}, i}(i \in\{1, \cdots, N\})$, are constant, which means that they can be obtained by measurements or designed in advance. Therefore, if given a closed form formulation of $\kappa_{\mathrm{q}, i}$ as a function of position, a circuit model that fully describes the QSCR channel can be obtained. It is known that the coupling coefficient $\kappa_{\mathrm{q}, i}$ can be formulated as below, using the amplitude of the total magnetic energy, $\alpha$, and the magnetic flux interlinking with the coil resonator, $\beta$ [5].

$$
\begin{aligned}
\kappa_{\mathrm{q}, i} & =\frac{\sqrt{2}}{4} \frac{\omega_{0} \beta_{\mathrm{q}, i}}{\sqrt{L_{i} \alpha_{\mathrm{q}}}} \\
\alpha_{\mathrm{q}} & =\iiint_{V_{\mathrm{q}}} \frac{\mu_{0}}{2}|\vec{H}|^{2} \mathrm{~d} V \\
\beta_{\mathrm{q}, i} & =\iint_{A_{i}} \mu_{0} \vec{H} \cdot \vec{n} \mathrm{~d} A
\end{aligned}
$$

$L_{i}, \mu_{0}, V$, and $A$ represent the inductance of coil resonator $i$, the permeability of vacuum, the volume of the QSCR, and the area enclosed by the coil resonator, respectively. We now assume a quasistatic circuit model in Figure 5a; based on circuit theory, the amplitude of the total magnetic energy $\alpha_{\mathrm{q}}$ is equal to the energy stored in the inductance of the QSCR [5]. Previous analysis of QSCR shows that the magnetic field $\vec{H}$ generated at $(r, \phi, z)$, using the cylindrical coordinates $(r, \phi, z)$ shown in Figure 1 (a), can be approximately expressed as Equation 5 [5].

$$
\vec{H}(r, \phi, z) \sim \frac{I_{0}}{2 \pi} \frac{1}{r} \vec{e}_{\phi}
$$

Here, $\vec{e}_{\phi}$ is a unit vector that rotates around the central conductive pole. It must be noted that former studies showed that the magnetic field has nearly no dependence in the $z$-axis (the direction parallel to the central pole), therefore $z$ doesn't appear in Equation 5. Since the scale of the end node and the QSCR is significantly different, it can be assumed that the magnetic flux that interlinks with the coil resonator is spatially uniform. Under these 
assumptions, $\alpha_{\mathrm{q}}$ and the approximate form of $\beta_{\mathrm{q}, i}$ can be formulated as follows:

$$
\begin{aligned}
\alpha_{\mathrm{q}} & =\frac{1}{2} L_{\mathrm{q}} I_{0}^{2} \\
\beta_{\mathrm{q}, i} & \sim \frac{\mu_{0} I_{0} s_{i}^{2} N_{i}}{2 r_{i}} \vec{e}_{\phi} \cdot \vec{e}_{n, i}
\end{aligned}
$$

$L_{\mathrm{q}}$ is the QSCR's inductance and $I_{0}$ is the amplitude of the total current flowing through the QSCR. $s_{i}$ and $N_{i}$ are the radius and the number of turns of the coil resonator $i$, respectively. $\vec{e}_{n, i}$ is the unit normal vector of the receiver coil resonator. Using Equations 2, 6, and 7, the coupling coefficient between the end node $i$ and the QSCR can be formulated as Equation 9.

$$
\begin{aligned}
\kappa_{\mathrm{q}, i}\left(r_{i}, \theta_{i}\right) & \sim \frac{\omega_{0} \mu_{0} N_{i} s_{i}^{2}}{4 \sqrt{L_{\mathrm{q}} L_{i}}} \frac{\vec{e}_{\phi} \cdot \vec{e}_{n, i}}{r_{i}} \\
& =\frac{\omega_{0} \mu_{0} N_{i} s_{i}^{2}}{4 \sqrt{L_{\mathrm{q}} L_{i}}} \frac{\cos \theta_{i}}{r_{i}}
\end{aligned}
$$

$\theta_{i}$ is the angle between the surface vector of the coil resonator $i$ and $\vec{e}_{\phi}$. Using this formulation, the full circuit model of the QSCR can be obtained.

Analysis as an $N$-port network. Next, to explore the characteristics of the QSCR as an $N$-port network, the forward voltage gain $\left(\left|S_{21}\right|\right)$ in the direction of (a) the central unit to an end node (or vice-versa, assuming duality) and (b) an end node to another end node, are analyzed. It is noted that $\left|S_{21}\right|^{2}$ also corresponds to the power transfer efficiency when no adaptive tuning mechanisms exist. The analytic formula when $N$ nodes exist in the room are shown in Appendix A [See Equations 13 and 14]; now that we have a simplified expression of all parameters in this equation, the characteristic of the link itself can be calculated using the position of the nodes.

To confirm the validity of these formulations and to obtain an intuitive sense of this QSCR channel, the calculated and measured forward voltage gains are also plotted in Figure 5b; the measured parameters shown on Table 1 were used in the calculated plot. The effects of other end nodes existing in the QSCR and the source impedance of the central unit were neglected in Figure $5 \mathrm{~b}$ for simplicity. It can be seen that the $3 \mathrm{~dB}$ voltage bandwidth of this channel is around 5 to $10 \mathrm{kHz}$ in this configuration for both cases.

Analysis for Wireless Power Transfer. The power transfer efficiency under conjugate impedance-matched conditions (i.e., maximum available power transfer efficiency) can be formulated as Equation 10, which can now be treated as a function of the positions of the end nodes by using Equation 9 [5].

$$
\begin{aligned}
G_{\max , i}\left(r_{i}, \theta_{i}\right) & =\frac{\chi_{i}\left(r_{i}, \theta_{i}\right)}{\left(1+\sqrt{1+\chi_{i}\left(r_{i}, \theta_{i}\right)}\right)^{2}} \\
\chi_{i}\left(r_{i}, \theta_{i}\right) & =\frac{4 Q_{\mathrm{q}} Q_{i}\left|\kappa_{\mathrm{q}, i}\left(r_{i}, \theta_{i}\right)\right|^{2}}{\omega_{0}^{2}} \\
& =\frac{\mu_{0}^{2}}{4} \cdot \underbrace{\frac{Q_{\mathrm{q}} Q_{i} N_{i}^{2} s_{i}^{4}}{L_{\mathrm{q}} L_{i}}}_{\text {fixed values }} \cdot \underbrace{\frac{\cos ^{2} \theta_{i}}{r_{i}^{2}}}_{\text {position }}
\end{aligned}
$$

$Q_{\mathrm{q}}$ and $Q_{i}$ represent the $Q$-factor of the QSCR and the coil resonator, respectively. Although this formulation does not consider the loss in the impedance tuning networks, former studies show that this corresponds well with actual measured results [5]. 


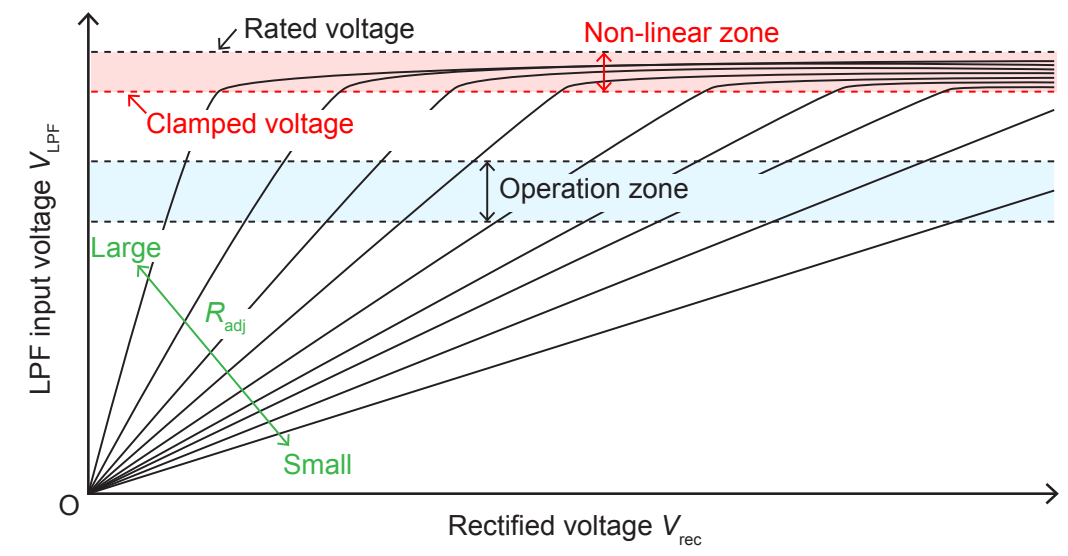

Fig. 6. Overview of the relationship between the rectified voltage $V_{\text {rec }}$ and the LPF input voltage $V_{\text {LPF }}$ [See Figure 4]. This diagram describes the signal level normalization stage. By changing the value of the digital potentiometer $R_{\text {adj }}$, the curves where the system operates can be changed.

\section{DESIGN AND IMPLEMENTATION OF THE END NODES}

The functions required for the end nodes are listed below.

- Receive power from the ambient magnetic field and charge the battery.

- Transmit data by modulating the ambient magnetic field and embedding the baseband signal.

- Receive data by sensing the ambient magnetic field and decoding the baseband signal.

The end nodes are designed so it executes these three functions above, one at a time. An alternative operation procedure is to simultaneously receive power and transmit/receive data, however, the difference here is subtle since we store the received power in a battery, which is a very practical setup. We selected the operation procedure that switches between the three above-mentioned functions in a time division manner, considering that it is beneficial to obtain fundamental results for both WPT/communication independently.

The studied system differs from typical wireless communication systems in two ways, which these differences bring about challenges: the large dynamic range of input signals and the narrow bandwidth of the QSCR channel. Also, since suppressing power consumption is one important objective of this study, an additional requirement is to use low-cost, low-power components. The large dynamic range of input signals occur because of three factors: (i) the operation is in near-field, (ii) the coupling state intensely changes, and (iii) power/data transfer links co-exist. This is a rather minor challenge in far-field systems and conventional near-field communication (NFC) systems since far-field systems have nearly static antenna impedance and operate at low power levels, whereas NFC doesn't assume large variations in receiver positions; this is a challenge specific to our system which needs to be solved for. The narrow bandwidth is a difficulty we need to overcome since high $Q$-factor is necessary for high-efficiency power transfer as shown in Equation 10, although $Q$-factor is inverse proportional to bandwidth [10]. In order to handle these issues, we custom designed a circuit which consists of a hardware/software-combined decoding circuit, power management unit, 3rd order LPF, and so on; the design of the end node is shown in Figure 4 and the implemented node is shown in Figure $3 \mathrm{~b}$. The tuning process of the impedance tuning network shown in Figure 4 is described in Section 5. In the remaining of this section, we further describe our design of each element.

Power management. The power management partition consists of a pre-regulator with high voltage tolerance, a Li-Po battery charger, a Li-Po battery, and a voltage regulator for the power supply of the entire end node. The $\mathrm{ON} / \mathrm{OFF}$ of the pre-regulator is controlled by the micro-controller of the node: ON when receiving power and 
(a) Efficiency with static impedance tuning

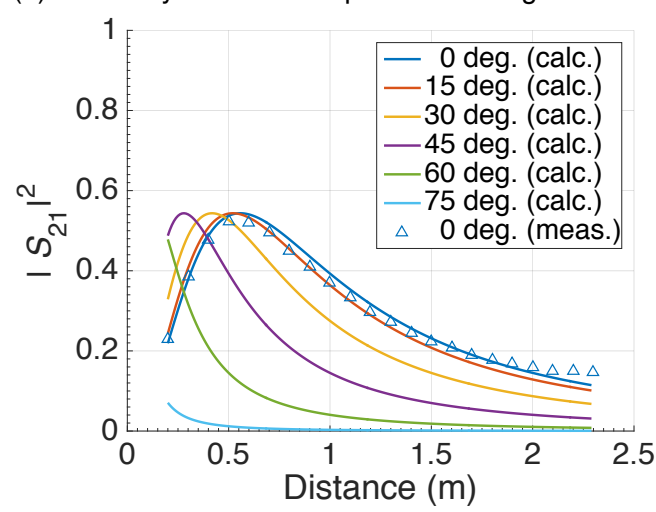

(b) Efficiency with adaptive impedance tuning

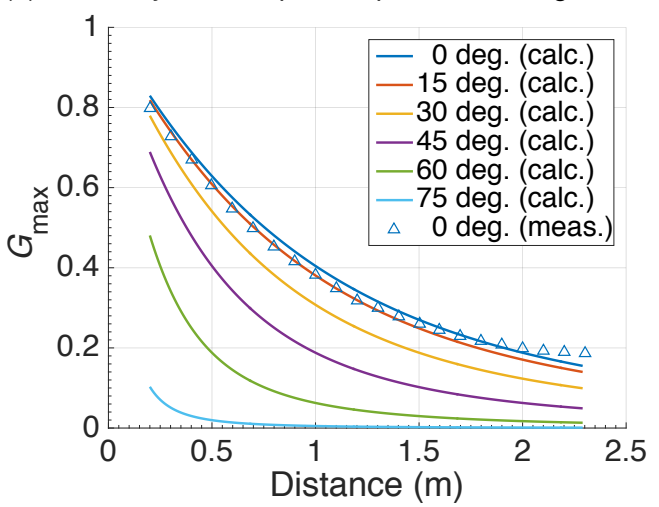

Fig. 7. Calculated and measured values of (a) forward power gain $\left|S_{21}\right|^{2}$ (i.e. WPT efficiency with static impedance tuning; Equation 13) and (b) maximum power transfer efficiency $G_{\max }$ (i.e. WPT efficiency with adaptive impedance tuning; Equation 10), when the position and the orientation of the end node is varied. The position refers to the distance from the central pole, whereas orientation refers to $\theta$ used in Equation 9, which is the angle between the surface vector of the receiver coil resonator and $\vec{e}_{\phi}$ [see Figure 1]. The parameters in Table 1 was used for the calculation. It can be seen that the measured values correspond well with calculated results.

OFF when receiving/transmitting data. When the pre-regulator is OFF, the impedance of the power management partition becomes sufficiently high and reduces interference with the data receiving/transmitting partition. The input impedance of the battery charger in constant output current (the state with maximum power consumption, since the battery charger operates with constant input voltage) was set to approximately $50 \Omega(5 \mathrm{~V}, 0.1 \mathrm{~A})$, which this impedance rises as the battery charger mitigates to constant output voltage operation. Therefore, considering that the input impedance of an ideal full-bridge rectifier is $\frac{8}{\pi^{2}} R_{l}\left(R_{l}\right.$ is the connected DC load), it should be a fair approximation to consider the whole system as a multiple port system with $50 \Omega$ port impedance, when the end node operates in power receiving mode.

Transmitting data. The end node transmits packets by switching on and off the N-MOSFET switches shown as "modulator" in Figure 4. When the gate voltage is high (e.g., the N-MOSFETs are on) the impedance is practically a short $(\sim 100 \mathrm{~m} \Omega)$ and when the gate voltage is low (e.g., the N-MOSFETs are off) the impedance is practically an open $(\sim 100 \mathrm{k} \Omega)$. This switching varies the reflected impedance seen from the QSCR and modulates the current and consequently the internal magnetic field of the QSCR [9].

Receiving data. The end node receives data by detecting the fluctuation of the ambient magnetic field caused by the central unit or another end node. Note that this is an asynchronous demodulation. The raw signal is received as fluctuations of the rectified voltage $V_{\text {rec }}$ [see Figure 4]. The dynamic range of this voltage $V_{\text {rec }}$ is extremely high in naively designed systems since this system works in near-field and the dynamic range of coupling coefficient is also wide. To handle this large dynamic range input with low-power low-cost components, we custom designed a hardware/software-combined signal decoder; this circuit dynamically adapts the incoming signal levels to a fixed range through software control, whereas the hardware partition protects the component from fast voltage fluctuations.

The rectified signal $V_{\text {rec }}$ is input to this decoder and passes through the signal level normalization partition, which consists of a peak detector, an adaptive voltage divider, and a voltage clamp as shown in Figure 4. This signal level normalization process is described in the following by using Figure 6, which shows the correspondence 


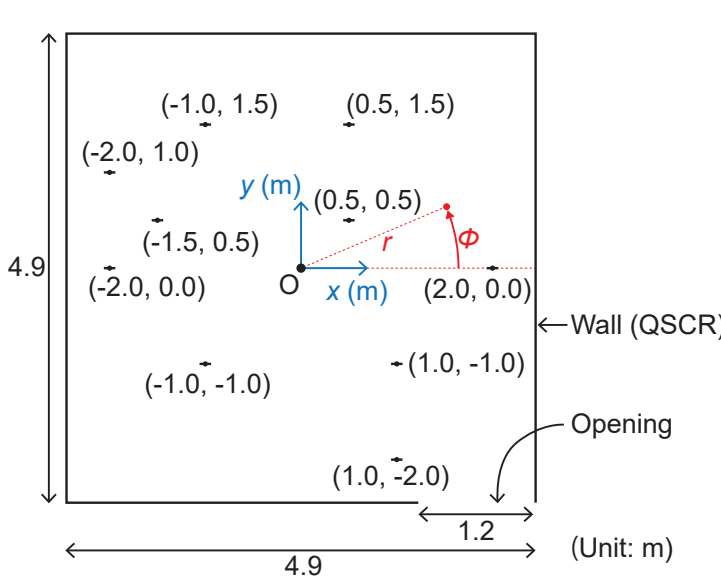

(a)

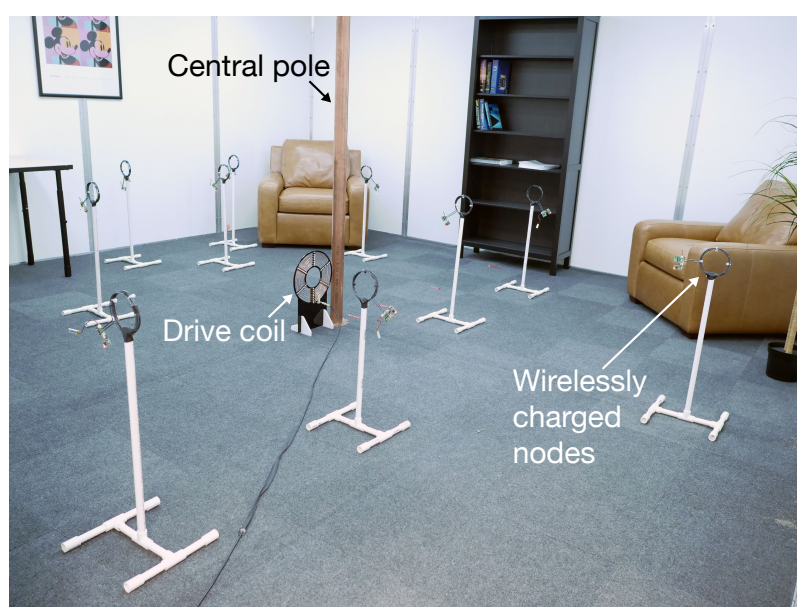

(b)

Fig. 8. (a) Placement of the end nodes in the wireless charging test. The orientations of the end nodes were set so that the surface vector of the end nodes were parallel to the $y$-axis. Point $\mathrm{O}$ represents the position of the central conductive pole. (b) Node placement in wireless charging test.

of the input voltage of the rectifier $V_{\text {rec }}$ and the input voltage of the low pass filter $V_{\text {LPF }}$ [see Figure 4]; as an overview, this signal level normalization function fulfills the three requirements below.

(1) Normalize the level of $V_{\mathrm{LPF}}$ to a fixed range.

(2) Regulate the signal level to protect the circuit components in later stages from fast voltage fluctuations.

(3) Prevent non-linear distortion that stems from the signal level regulation.

The signal level is sensed by a peak detector and $R_{\text {adj }}$ [see Figure 4] is iteratively adjusted so that the output of the peak detector tracks a fixed voltage range. This corresponds to selecting the appropriate curves in Figure 6. Although, with only software control which is comparatively slow, $V_{\mathrm{LPF}}$ easily exceeds the rated voltage of the latter circuit; we need hardware mechanisms to prevent this. Therefore, we added a steep voltage clamp consisting of a Zener diode and a bipolar junction transistor (BJT) to clamp the LPF input voltage $V_{\mathrm{LPF}}$ [see Figure 4]. However, this brings about another problem; with the voltage clamped in a non-linear manner at some level, the signals around the clamped level also get clamped (i.e., distorted). To prevent this distortion $R_{\text {adj }}$ is controlled so the operation zone is pinned far enough from the non-linear zone [see Figure 4]. This normalized signal passes through a 3rd order active LPF, which results in a smoothed output with the carrier frequency and the harmonic compositions cut off. (Note that this is a asynchronous demodulation.) This sharp filtering is necessary since the bandwidth of the channel is narrow throughout this study. This LPF gives an output with baseband signals standing out. These baseband signals are transformed into digital signals using a comparator; this is executed by comparing the direct LPF output and the LPF output with an additional large time constant averaging [24].

\section{EVALUATION OF POWER TRANSFER}

The power transfer capabilities from the QSCR to the end nodes were evaluated through numerical analysis, circuit simulations, and measurements. First, the parameters of the impedance tuning network were determined 
through circuit simulations using Keysight ADS [42]. The values $\kappa_{\mathrm{L} i, i}$ and $\kappa_{\mathrm{s}, \mathrm{q}}$ shown in Figure 5a was the two parameters to be determined. These two values were swept to maximize the average $\left|S_{21}\right|^{2}$ value (i.e., the power transfer efficiency when a $50 \Omega$ power source and $50 \Omega$ load is used) when the position of the end node moves from $x=0.2 \mathrm{~m}$ (next to the central pole) to $x=2.3 \mathrm{~m}$ (next to the wall) in an orientation perpendicular to the magnetic flux [5]. It was assumed that only one end node exists inside the QSCR and no dynamic impedance tuning is implemented. The port impedance of both nodes was set to $50 \Omega$, considering the system design explained in Section 4. The $Q$-factors of the drive coil, the QSCR, and the end node resonators were 630, 1670, and 220, respectively. The obtained optimal $\kappa_{\mathrm{L} i, i}$ and $\kappa_{\mathrm{s}, \mathrm{q}}$ values were physically implemented by adjusting the position of the drive coil and load loop represented as $L_{\mathrm{s}}$ and $L_{\mathrm{L} m}$ in Figure $5 \mathrm{a}$. Next, the $\left|S_{21}\right|^{2}$ values (i.e. transfer efficiency of $50 \Omega$ systems with static impedance tuning) and the maximum available efficiency $G_{\max }$ (i.e. transfer efficiency with adaptive impedance tuning) were measured in various positions using a vector network analyzer [48]. The measured and calculated results are shown in Figure 7 and it can be seen that the measured results correspond with the calculated results very well. This calculated result was obtained by using Equations 13 and 10. It can be seen that the transfer efficiency with static tuning $\left(\left|S_{21}\right|^{2}\right)$ doesn't increase monotonically as distance decreases, but shows a peak at a specific distance [See figure 7(a)]. This is because the two resonators become "over-coupled" when placed closer than a specific distance, which increases the return loss of the power source; it can also be seen that this phenomenon can be overcome by adopting adaptive impedance tuning techniques or using switching power sources [See figure 7(b)]. In addition to the measurements of power transfer to a single end node, the power transfer in a more realistic scenario was tested. Ten end nodes were implemented and placed inside the QSCR room as shown in Figure 8a. The setup of this test is shown in Figure 8b and some extra furniture (sofa, table, bookshelf, etc) were placed in the QSCR to emulate realistic scenarios. Through this test, it was confirmed that all of the end nodes were able to be simultaneously charged (i.e., all battery charger ICs turned in to charging mode) with a $20 \mathrm{~W}$ power input from the central unit. It is noted that since all ten nodes have non-linear load impedance, which varies depending on the battery state and input voltage, the system was not necessarily in impedance-matched conditions.

\section{EVALUATION OF DATA TRANSFER}

To evaluate the system performance, we measure the Bit Error Rate (BER) in three communication links: (a) end node to end node (Node-to-Node), and (b) central unit to end node (Central-to-Node) and (c) end node to central unit (Node-to-Central). For these three different links, the communication performance of the whole system was evaluated through measurements at various distances, orientations, and data rates.

\subsection{Bit Error Rate Measurements and Performance Evaluation}

Evaluation setup. The data is transmitted by using a packet which contains 5 bits preamble and 20 bits data payload with 1-to-50 kbps throughput. The BER measurement is done by calculating the errors occurring in 1000 received bits. If the corresponding preambles are not found in any of the received bits, then all the 1000 bits are viewed as errors such that the BER is 1 . Otherwise, the errors are calculated by accumulating the errors in data payload and the total unknown bits, which no preambles can be found out for extracting data payloads.

Distance/orientation vs BER test procedure. For the node-to-node test, the SDR only generates a $1.325 \mathrm{MHz}$ constant amplitude sine wave to generate the magnetic field channel in the QSCR. Using the $x y$ coordinates shown in Figure 8a, an end node which acts as a transmitter is placed on the $y$-axis, $1 \mathrm{~m}$ away from the central conductive pole; the end node which acts as a receiver is placed on the $x$-axis, with the distance from the pole varied from $25 \mathrm{~cm}$ to $225 \mathrm{~cm}$ with a $25 \mathrm{~cm}$ interval. We also vary the orientation of the end nodes at each location to evaluate the orientation dependency. For both Node-to-Central and Central-to-Node evaluation tests, the central unit transmits and receives data through the QSCR channel while an end node is placed on the $x$-axis 


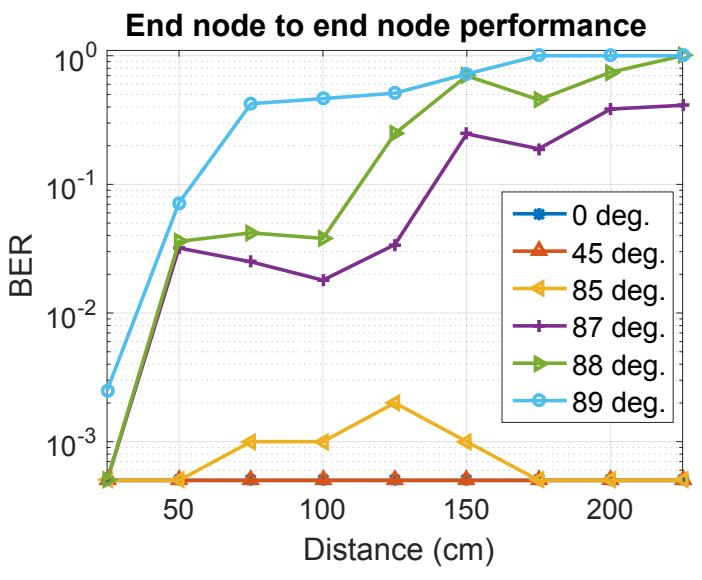

(a)

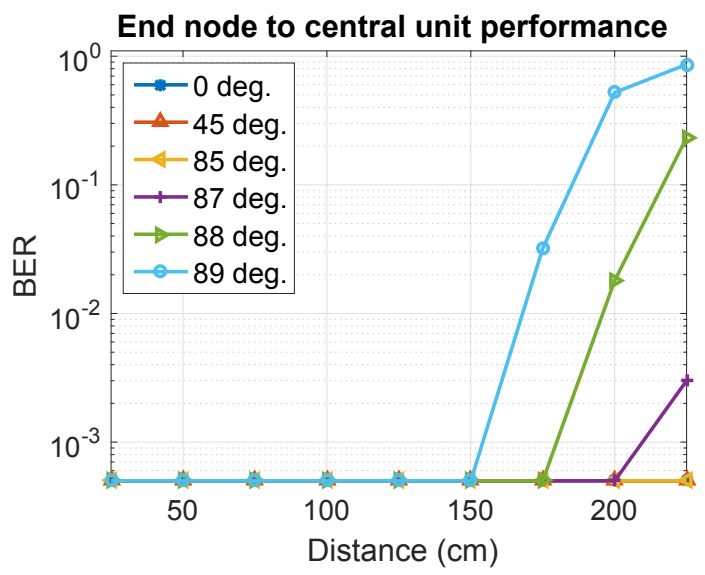

(c)

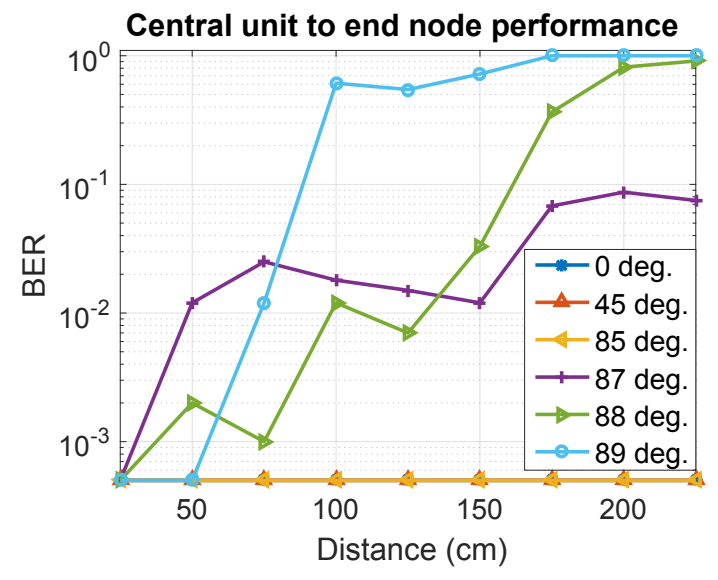

(b)

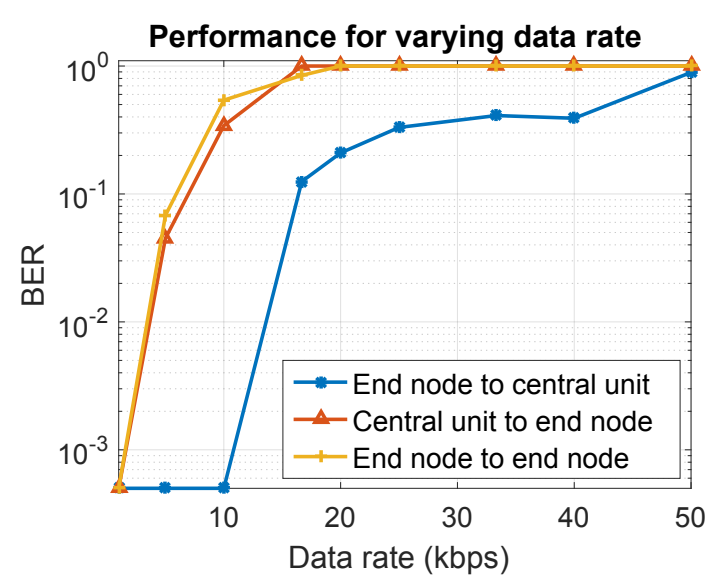

(d)

Fig. 9. Evaluations of the system performance through different distance, orientation and data rate. The orientation here refers to $\theta$ used in Equation 9, which is the angle between the surface vector of the coil resonator and $\vec{e}_{\phi}$ [see Figure 8b]. Panel (a) shows end node to end node communication, with the location and orientation of the receiver varied. Panels (b) and (c) show central unit to end node and end node to central unit communication performance by varying the distance and orientation. Panel (d) shows the system performance under different data rates, for the three data transfer links, with fixed end node positions.

with different distances and orientations. It should be noted that for the node-to-central communication test, the SDR only transmits constant power, although, for the central-to-node test, the SDR provides both power and data through binary ASK modulation.

Data rate vs BER test procedure. In addition to applying different distances and orientations for evaluation, we test the performance of three communication links by adopting different data rates from $1 \mathrm{kbps}$ to $50 \mathrm{kbps}$. The node-to-node tests were executed with an end node which acts as a transmitter, placed on the $y$-axis, $1 \mathrm{~m}$ 


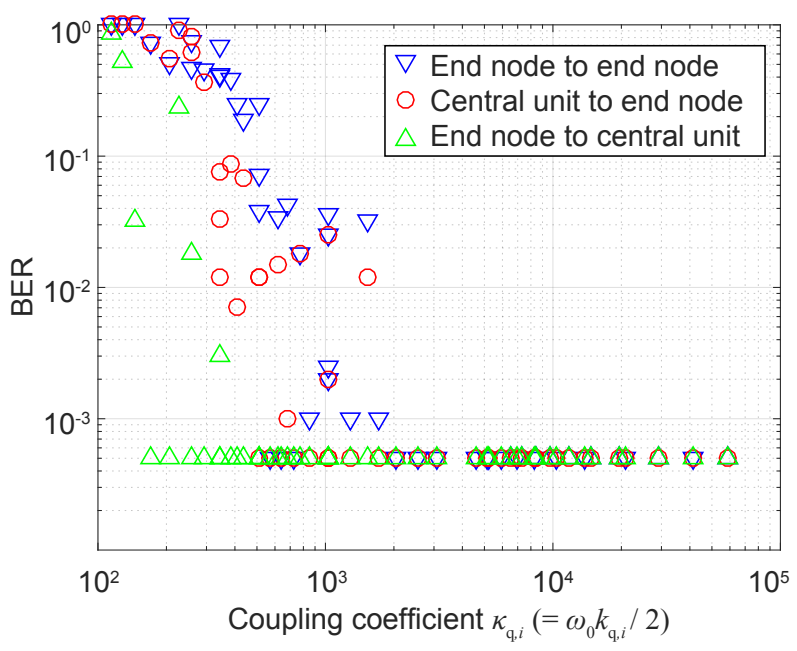

Fig. 10. Figures 9a, 9b, and 9c re-plotted to the relationship between coupling coefficient $\kappa_{\mathrm{q}, \mathrm{i}}$ [see Equation 2] and BER.

away from the central pole, while another end node acting as a receiver was placed on the $x$-axis, also $1 \mathrm{~m}$ away from the central pole. For both node-to-central and central-to-node tests, an end node was placed on the $x$-axis, $1 \mathrm{~m}$ away from the central pole. It is noted that the end nodes were placed in perfect orientation (orthogonal to the ambient magnetic field vector) in this series of measurements to evaluate various data rate alone.

Results and insights. Figure 9a shows the node-to-node communication performance with distance and orientation varied. The orientation here refers to $\theta$ used in Equation 9, which is the angle between the surface vector of the coil resonator and $\vec{e}_{\phi}$ [see Figure 8b]. As Equation 9 shows, the coupling coefficient $\kappa_{\mathrm{q}, i}$ is maximized when $\theta$ is 0 degree and is zero when $\theta$ is 90 degrees. A higher coupling coefficient means that the end node can receive and modulate the $1.325 \mathrm{MHz}$ carrier for data transfer efficiently. When the orientation $\theta$ is set to 0 or 45 degrees, the end node receiver does not have any errors. When the orientation $\theta$ is over 85 degrees, the error starts to occur. When $\theta$ is near 90 degree, such as 89 degree, the BER increases significantly. In addition to orientation, when the distance increases, the received carrier signals at the end node also decreases [see Equation 9]. Under this situation, the load modulation can only modulate the tiny carrier signals, therefore, the errors increase along with the distance from the central pole. Similarly, in Figure $9 \mathrm{~b}$ and $9 \mathrm{c}$, longer distance and larger $\theta$ results in higher BER. Also, it can be noticed that the node-to-central link has better performance compared to the node-to-node link when given the same distance and orientation. This is because SDR is a very high-end receiver compared to the low-cost end node receiver.

Figure $9 \mathrm{~d}$ shows the BER performance when varying the data rate. Since the $3 \mathrm{~dB}$ bandwidth of the channel in the QSCR room with this configuration is around 5 to $10 \mathrm{kHz}$ as shown in Figure 5b, we expect that the performance degrades when data rate exceeds around $5 \mathrm{kHz}$. However, for the node-to-central communication link, the system can operate with no errors up to $10 \mathrm{kbps}$. This is also due to the high sensitivity of the X310 SDR. The other two links have similar performance and the error starts to occur when the data rate is over $5 \mathrm{kHz}$. Thus, the $1.325 \mathrm{MHz}$ channel bandwidth shown in Figure 5b plays an important role to estimate maximal obtainable data rate.

Figure 9a,9b, and 9c, have showed that both distance and orientation plays an important role in BER performance. Since this system operates in near-field, these two parameters can be combined and considered as a single metric when it is converted into coupling coefficient via Equation 9. The results when the distance and orientation are 
converted into coupling coefficient $\kappa$ is shown in Figure 10. When the distance or orientation $\theta$ increases, the coupling coefficient $\kappa_{\mathrm{q}, i}$ decreases. Obviously, it can be seen that the BER is lower when the coupling coefficient is higher.

In this study, the orientation was an issue since we used a single coil resonator in the end nodes. To overcome this, we can combine three orthogonal coil resonators as shown in previous work [5], which should result in orientation-independent, high-quality communication.

\section{CONCLUSIONS AND FUTURE WORKS}

In this paper, the use of QSCR as a data and power transfer link was explored. The QSCR channel was analyzed and evaluated for both power and data transfer means, through circuit analysis and measurements. Then, an actual QSCR and load-modulation based power/data transfer system was implemented and the system was evaluated from both power/data transfer aspects. Through experiments of power transfer, it was shown that it is possible to simultaneously charge 10 end nodes placed in realistic scenarios. Through BER measurements, it was shown that the high-quality communication links can be established in three directions: end node to end node, end node to central unit, and central unit to end node. Below we raise some possibilities for future improvement.

Development of the MAC layer protocols. Our study focused on the physical layer of the system, although considerations must be also made for the MAC layer to optimize communication and power distribution as a whole system [2]. In the future, we will adopt time-division multiplexing methods on the MAC layer for integrating a large number of end nodes into a single system $[9,46]$. Ultimately, with time-multiplexing protocols, our system enables the preferred end nodes for providing both WPT and data communication purpose.

Dynamic impedance tuning. Although we believe that our static tuning networks are satisfactory for powering low-power nodes (around a Watt), assuming the charging of many higher power consumption (over ten watts) nodes, it is beneficial to introduce dynamic tuning to aim for the physical upper limit of efficiency. Integrating maximum efficiency point tracking (MEPT) mechanisms based on dynamic impedance conversion methods should be one promising direction of future work [29, 33].

Enhancing the data rate. Our study focuses on the physical layer of the system and only assume low-cost, low-power hardware since we believe this is one of the most necessary missing pieces of IoT. Consequently, the data rates in this study were limited to a few $\mathrm{kHz}$, which is quite satisfactory for many standard functions.

Although it is out of scope for this study, there are many applications such as video streaming that require much higher data rates; another possible research direction should be applying advanced modulation techniques (e.g. PSK, QAM, etc) and employing rich hardware (e.g., SDR) on both RX/TX sides, and examine the upper limit of data that can be transferred through the QSCR channel.

\section{APPENDIX}

\section{A ANALYTIC FORWARD VOLTAGE GAIN OF THE QSCR-BASED LINKS}

An analytic expression of the $N$-port system circuit model shown in Figure $5 \mathrm{a}$ is provided in this appendix. The forward voltage gain $\left(S_{21}\right)$ in the direction of (a) the central unit to an end node (or vice-versa) and (b) an end node to another end node, are calculated. The analytic formulation when $N$ end nodes exist in the room are shown in Equation 13 and 14. It is noted that the port impedance $Z_{0}$ was assumed to be uniform for all measured ports. 


$$
\begin{aligned}
S_{m, \text { central }} & =\frac{2 Z_{\mathrm{s}, \mathrm{q}} Z_{\mathrm{q}, m} Z_{\mathrm{L} m, m} Z_{0}}{\left\{\left(Z_{\mathrm{s}}+Z_{0}\right) A+\left|Z_{\mathrm{s}, \mathrm{q}}\right|^{2}\right\}\left\{Z_{m}\left(Z_{\mathrm{L} m}+Z_{0}\right)+\left|Z_{\mathrm{L} m, m}\right|^{2}\right\}+\left|Z_{\mathrm{q}, m}\right|^{2}\left(Z_{\mathrm{s}}+Z_{0}\right)\left(Z_{\mathrm{L} m}+Z_{0}\right)} \\
S_{m, n} & =\frac{2 Z_{\mathrm{L} m, m} Z_{\mathrm{q}, m} Z_{\mathrm{q}, n} Z_{\mathrm{L}, n} Z_{0}}{\left(D_{m}+Z_{0} Z_{m}\right) E_{n}+\left(D_{n}+Z_{0} Z_{n}\right) E_{m}+B\left(D_{m} D_{n}-Z_{0}^{2} Z_{m} Z_{n}\right)} \\
Z_{X} & \left.=R_{X}+j \omega_{0} L_{X}+\frac{1}{\left(j \omega_{0} C_{X}\right)} \quad\left(X \in\left\{\mathrm{s}, \mathrm{q}, 1, \cdots, i, \cdots, N, \mathrm{~L}_{1}, \cdots, \mathrm{L}_{i}, \cdots, \mathrm{L}_{N}\right\}\right\}\right) \\
Z_{X, Y}=j 2 \kappa_{X, Y} \sqrt{L_{X} L_{Y}}\left(X \neq Y ; \quad X, Y \in\left\{\mathrm{s}, \mathrm{q}, 1, \cdots, i, \cdots, N, \mathrm{~L}_{1}, \cdots, \mathrm{L}_{i}, \cdots, \mathrm{L}_{N}\right\}\right) & \\
A & =\sum_{i \neq m}^{N}\left[\frac{\left|Z_{\mathrm{q}, i}\right|^{2}}{\left.\left\{Z_{i}+\frac{\left|Z_{\mathrm{L} i, i}\right|^{2}}{Z_{\mathrm{L}, i}+Z_{\mathrm{Load}, i}}\right\}\right]+Z_{\mathrm{q}},}\right. \\
B & =\sum_{i \neq m, n}^{N}\left[\frac{\left|Z_{\mathrm{q}, i}\right|^{2}}{\left\{Z_{i}+\frac{\left|Z_{\mathrm{L}, i, i}\right|^{2}}{Z_{\mathrm{L}, i}+Z_{\mathrm{Load}, i}}\right\}}\right]+\frac{\left|Z_{\mathrm{s}, \mathrm{q}}\right|^{2}}{Z_{\mathrm{S}}+Z_{\mathrm{in}}}+Z_{\mathrm{q}} \\
D_{i} & =Z_{i} Z_{\mathrm{L} i}+\left|Z_{\mathrm{L} i, i}\right|^{2} \\
E_{i} & =\left\{\left|Z_{\mathrm{q}, i}\right|^{2}\left(Z_{\mathrm{L} i}+Z_{0}\right)+Z_{i} Z_{0} B\right\}
\end{aligned}
$$

\section{B THE EFFECT OF FOREIGN METALLIC OBJECTS}

QSCR generates a quasi-static field distribution, not a far-field propagating wave; therefore multi-path interference as typically affects Wi-Fi and cellular signals do not occur in this system. The QSCR chamber operates in the deep sub-wavelength region, which the wavelength of the $1.325 \mathrm{MHz}$ carrier is approximately $230 \mathrm{~m}$ in free space, whereas the size of the room is only $4.9 \mathrm{~m} \times 4.9 \mathrm{~m} \times 2.3 \mathrm{~m}$; this means the magnetic field distribution is effectively "static". The magnetic field is not beamed from one point to another nor is it pointed at the target. Instead, the flow of current through the walls, floor, and ceiling generates the field at all points within the cavity volume. Thus, if a metallic object is placed in the QSCR room, the magnetic field forms around the object. This can be seen in figure 11, which shows a COMSOL simulation of the QSCR room with a 15-inch MacBook Pro, modeled as a solid block of aluminum, placed in halfway from the pole to the wall with the worst-orientation [see figure 11 (a)]. Figure 11 (b) shows the magnetic field distribution (top view) with arrows showing direction and color indicating the magnitude of the field strength. It can be seen the overall magnetic field distribution (which is directly correlated to power transfer and communication rate) is generally unaffected by the presence of the laptop. The detailed view in figure 11 (c) shows that indeed the magnetic field generated by the QSCR room is formed around the boundary condition of the laptop with the magnetic flux "flowing" around it. Further simulation results show that the Q-factor and the effective inductance of the QSCR decrease by less than $1 \%$ which is within the margin of error of this finite element simulation and the extremely high Q-factor of the QSCR.

Given the insight that the magnetic field within the QSCR "flows" around conductive objects, we can consider the edge conditions when the metallic objects become very large or very numerous. Consider the hypothetical case where the MacBook Pro shown in Figure 11 (a) is increased in size. As it gets much much larger than a bookshelf or a refrigerator, it will begin to inhabit a significant portion of the space between the pole and the 
(a) Simulation model

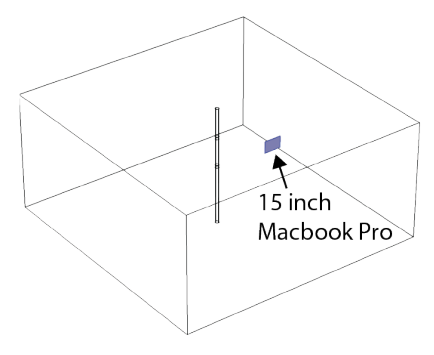

(b) Top view of H-field

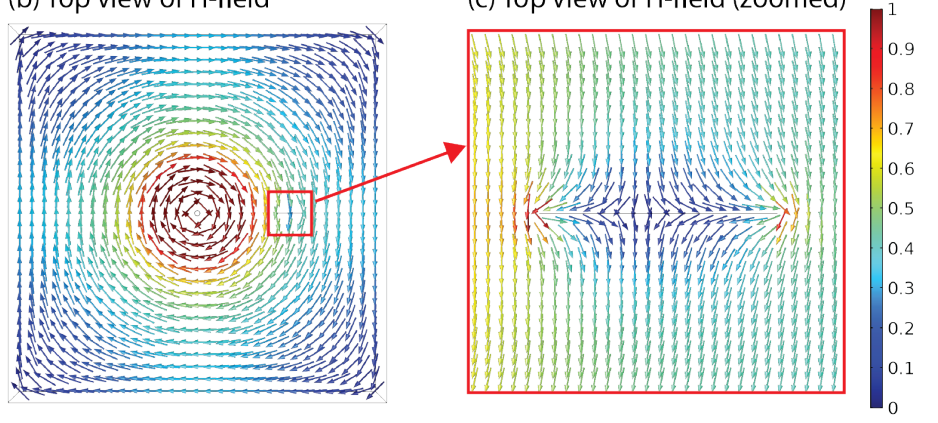

Fig. 11. FEM-based simulation for observing the effect of foreign metallic objects present in the QSCR channel. (a) Simulation setup. The Macbook pro is modeled as a solid sheet of aluminum. (b, c) Top view of the simulated magnetic field. (c) is a zoomed view of (b). It can be seen that the magnetic flux flows around metallic objects.

wall. This will begin to block the flux like a Faraday cage and alter the behavior of the QSCR room, changing the resonant frequency and possibly creating alternate resonant modes. In the limit as the hypothetical MacBook Pro grows to consume all the space, the room will no longer resonate. However, we consider this as an extreme edge condition as people do not typically bring huge plates of sheet metal into their homes.

\section{ACKNOWLEDGMENTS}

The authors would like to thank the reviewers for their insightful review comments. We would also like to thank Alexandra Delazio, William Hedberg, Ken Nakagaki, Dingtian Zhang, and Yang Zhang for their invaluable feedback throughout the project. This work was supported by a Grant-in-Aid for JSPS Fellows JP18J22537 and JST ERATO Grant Number JPMJER1501.

\section{REFERENCES}

[1] Luigi Atzori, Antonio Iera, and Giacomo Morabito. 2010. The Internet of Things: A survey. Computer Networks 54, 15 (October 2010), 2787 - 2805. https://doi.org/10.1016/j.comnet.2010.05.010

[2] Suzhi Bi, Chin Keong Ho, and Rui Zhang. 2015. Wireless powered communication: Opportunities and challenges. IEEE Communications Magazine 53, 4 (April 2015), 117-125. https://doi.org/10.1109/MCOM.2015.7081084

[3] William C Brown. 1984. The history of power transmission by radio waves. IEEE Transactions on Microwave Theory and Techniques 32, 9 (September 1984), 1230-1242. https://doi.org/10.1109/TMTT.1984.1132833

[4] Matthew J Chabalko and Alanson P Sample. 2014. Resonant cavity mode enabled wireless power transfer. Applied Physics Letters 105, 24 (December 2014), 243902. https://doi.org/10.1063/1.4904344

[5] Matthew J Chabalko, Mohsen Shahmohammadi, and Alanson P Sample. 2017. Quasistatic Cavity Resonance for Ubiquitous Wireless Power Transfer. PloS ONE 12, 2 (February 2017), e0169045. https://doi.org/10.1371/journal.pone.0169045

[6] Andreas Christ, Mark G Douglas, John M Roman, Emily B Cooper, Alanson P Sample, Benjamin H Waters, Joshua R Smith, and Niels Kuster. 2013. Evaluation of wireless resonant power transfer systems with human electromagnetic exposure limits. IEEE Transactions on Electromagnetic compatibility 55, 2 (April 2013), 265-274. https://doi.org/10.1109/TEMC.2012.2219870

[7] Joshua F Ensworth and Matthew S Reynolds. 2017. BLE-Backscatter: Ultralow-Power IoT Nodes Compatible With Bluetooth 4.0 Low Energy (BLE) Smartphones and Tablets. IEEE Transactions on Microwave Theory and Techniques 65, 9 (Sept 2017), $3360-3368$. https://doi.org/10.1109/TMTT.2017.2687866

[8] GS1 EPCglobal Inc. 2015. EPC UHF Gen2 Air Interface Protocol. (April 2015). https://www.gs1.org/standards/epc-rfid/ uhf-air-interface-protocol/2-0-1 (Accessed on May 15, 2018).

[9] Klaus Finkenzeller. 2010. RFID handbook: fundamentals and applications in contactless smart cards, radio frequency identification and near-field communication. John Wiley \& Sons. 
[10] Pulkit Grover and Anant Sahai. 2010. Shannon meets Tesla: Wireless information and power transfer. In Proceedings of the 2010 IEEE International Symposium on Information Theory (ISIT). IEEE, Austin, Texas, U.S.A., 2363-2367. https://doi.org/10.1109/ISIT.2010.5513714

[11] Hermann A Haus and Weiping Huang. 1991. Coupled-mode theory. Proc. IEEE 79, 10 (October 1991), 1505-1518. https://doi.org/10. $1109 / 5.104225$

[12] Shu Yuen Ron Hui, Wenxing Zhong, and Chi Kwan Lee. 2014. A critical review of recent progress in mid-range wireless power transfer. IEEE Transactions on Power Electronics 29, 9 (September 2014), 4500-4511. https://doi.org/10.1109/TPEL.2013.2249670

[13] Vikram Iyer, Elyas Bayati, Rajalakshmi Nandakumar, Arka Majumdar, and Shyamnath Gollakota. 2018. Charging a Smartphone Across a Room Using Lasers. Proceedings of the ACM on Interactive, Mobile, Wearable and Ubiquitous Technologies (IMWUT) 1, 4, Article 143 (Jan. 2018), 21 pages. https://doi.org/10.1145/3161163

[14] Hamid Jabbar, Young S Song, and Taikyeong Ted Jeong. 2010. RF energy harvesting system and circuits for charging of mobile devices. IEEE Transactions on Consumer Electronics 56, 1 (February 2010), 247-253. https://doi.org/10.1109/TCE.2010.5439152

[15] Jouya Jadidian and Dina Katabi. 2014. Magnetic MIMO: How to Charge Your Phone in Your Pocket. In Proceedings of the 20th Annual International Conference on Mobile Computing and Networking (MobiCom '14). ACM, New York, NY, USA, 495-506. https: //doi.org/10.1145/2639108.2639130

[16] Darko Kajfez and Eugene J Hwan. 1984. Q-Factor Measurement with Network Analyzer. IEEE Transactions on Microwave Theory and Techniques 32, 7 (July 1984), 666-670. https://doi.org/10.1109/TMTT.1984.1132751

[17] Salil Kashyap, Emil Björnson, and Erik G Larsson. 2015. Can wireless power transfer benefit from large transmitter arrays?. In Proceedings of the 2015 IEEE Wireless Power Transfer Conference (WPTC). 1-3. https://doi.org/10.1109/WPT.2015.7140132

[18] Bryce Kellogg, Aaron Parks, Shyamnath Gollakota, Joshua R. Smith, and David Wetherall. 2014. Wi-fi Backscatter: Internet Connectivity for RF-powered Devices. SIGCOMM Comput. Commun. Rev. 44, 4 (Aug. 2014), 607-618. https://doi.org/10.1145/2740070.2626319

[19] Mehdi Kiani and Maysam Ghovanloo. 2012. The Circuit Theory Behind Coupled-Mode Magnetic Resonance-Based Wireless Power Transmission. IEEE Transactions on Circuits and Systems I: Regular Papers 59, 9 (Sept 2012), 2065-2074. https://doi.org/10.1109/TCSI 2011.2180446

[20] Sangkil Kim, Rushi Vyas, Jo Bito, Kyriaki Niotaki, Ana Collado, Apostolos Georgiadis, and Manos M Tentzeris. 2014. Ambient RF Energy-Harvesting Technologies for Self-Sustainable Standalone Wireless Sensor Platforms. Proc. IEEE 102, 11 (Nov 2014), 1649-1666. https://doi.org/10.1109/JPROC.2014.2357031

[21] Ilkka Laakso, Shogo Tsuchida, Akimasa Hirata, and Yoshitsugu Kamimura. 2012. Evaluation of SAR in a human body model due to wireless power transmission in the $10 \mathrm{MHz}$ band. Physics in Medicine \& Biology 57, 15 (July 2012), 4991. https://doi.org/10.1088/ 0031-9155/57/15/4991

[22] Hans-Dieter Lang, Alon Ludwig, and Costas D Sarris. 2014. Convex optimization of wireless power transfer systems with multiple transmitters. IEEE Transactions on Antennas and Propagation 62, 9 (July 2014), 4623-4636. https://doi.org/10.1109/TAP.2014.2330584

[23] Xu Li, Rongxing Lu, Xiaohui Liang, Xuemin Shen, Jiming Chen, and Xiaodong Lin. 2011. Smart community: an internet of things application. IEEE Communications Magazine 49, 11 (Nov. 2011), 68-75. https://doi.org/10.1109/MCOM.2011.6069711

[24] Vincent Liu, Aaron Parks, Vamsi Talla, Shyamnath Gollakota, David Wetherall, and Joshua R Smith. 2013. Ambient backscatter: wireless communication out of thin air. ACM SIGCOMM Comput. Commun. Review 43, 4 (October 2013), 39-50. https://doi.org/10.1145/2534169. 2486015

[25] Xiao Lu, Ping Wang, Dusit Niyato, Dong In Kim, and Zhu Han. 2015. Wireless Networks With RF Energy Harvesting: A Contemporary Survey. IEEE Communications Surveys Tutorials 17, 2 (Secondquarter 2015), 757-789. https://doi.org/10.1109/COMST.2014.2368999

[26] Andrea Massa, Giacomo Oliveri, Federico Viani, and Paolo Rocca. 2013. Array Designs for Long-Distance Wireless Power Transmission: State-of-the-Art and Innovative Solutions. Proc. IEEE 101, 6 (June 2013), 1464-1481. https://doi.org/10.1109/JPROC.2013.2245491

[27] Henry Mei, Kyle A Thackston, Rebecca A Bercich, John GR Jefferys, and Pedro P Irazoqui. 2017. Cavity resonator wireless power transfer system for freely moving animal experiments. IEEE Transactions on Biomedical Engineering 64, 4 (April 2017), 775-785. https://doi.org/10.1109/TBME.2016.2576469

[28] Daniele Miorandi, Sabrina Sicari, Francesco De Pellegrini, and Imrich Chlamtac. 2012. Internet of Things. Ad Hoc Netw. 10, 7 (Sept. 2012), 1497-1516. https://doi.org/10.1016/j.adhoc.2012.02.016

[29] Jongmin Park, Youndo Tak, Yoongoo Kim, Youngwook Kim, and Sangwook Nam. 2011. Investigation of adaptive matching methods for near-field wireless power transfer. IEEE Transactions on Antennas and Propagation 59, 5 (March 2011), 1769-1773. https://doi.org/10. 1109/TAP.2011.2123061

[30] Ragunathan Raj Rajkumar, Insup Lee, Lui Sha, and John Stankovic. 2010. Cyber-physical systems: the next computing revolution. In Proceedings of the 47th design automation conference. ACM, 731-736. https://doi.org/10.1145/1837274.1837461

[31] David S Ricketts, Matthew J Chabalko, and Andrew Hillenius. 2013. Experimental demonstration of the equivalence of inductive and strongly coupled magnetic resonance wireless power transfer. Applied Physics Letters 102, 5 (January 2013), 053904. https: //doi.org/10.1063/1.4788748

[32] Alanson P Sample, David T Meyer, and Joshua R Smith. 2011. Analysis, experimental results, and range adaptation of magnetically coupled resonators for wireless power transfer. IEEE Transactions on Industrial Electronics 58, 2 (March 2011), 544-554. https:

Proc. ACM Interact. Mob. Wearable Ubiquitous Technol., Vol. 2, No. 4, Article 188. Publication date: December 2018. 
//doi.org/10.1109/TIE.2010.2046002

[33] Alanson P Sample, Benjamin H Waters, Scott T Wisdom, and Joshua R Smith. 2013. Enabling Seamless Wireless Power Delivery in Dynamic Environments. Proc. IEEE 101, 6 (June 2013), 1343-1358. https://doi.org/10.1109/JPROC.2013.2252453

[34] Alanson P Sample, Daniel J Yeager, Pauline S Powledge, Alexander V Mamishev, and Joshua R Smith. 2008. Design of an RFID-based battery-free programmable sensing platform. IEEE Transactions on Instrumentation and Measurement 57, 11 (June 2008), 2608-2615. https://doi.org/10.1109/TIM.2008.925019

[35] Takuya Sasatani, Matthew J Chabalko, Yoshihiro Kawahara, and Alanson P Sample. 2017. Multimode Quasistatic Cavity Resonators for Wireless Power Transfer. IEEE Antennas Wireless Propagation Letters 16 (August 2017), 2746-2749. https://doi.org/10.1109/LAWP.2017. 2744658

[36] Takuya Sasatani, Alanson P. Sample, and Yoshihiro Kawahara. 2018. 3-D Wireless Charging for Indoor Electronics Using Multimode Quasistatic Cavity Resonators. In Proceedings of the 2018 ACM International foint Conference and 2018 International Symposium on Pervasive and Ubiquitous Computing and Wearable Computers (UbiComp '18). ACM, 444-447. https://doi.org/10.1145/3267305.3267571

[37] Tsuyoshi Sekitani, Makoto Takamiya, Yoshiaki Noguchi, Shintaro Nakano, Yusaku Kato, Takayasu Sakurai, and Takao Someya. 2007. A large-area wireless power-transmission sheet using printed organic transistors and plastic MEMS switches. Nature materials 6, 6 (2007), 413. https://doi.org/doi:10.1038/nmat1903

[38] Naoki Shinohara. 2011. Power without wires. IEEE Microwave Magazine 12, 7 (December 2011), S64-S73. https://doi.org/10.1109/MMM. 2011.942732

[39] Mingzhao Song, Pavel Belov, and Polina Kapitanova. 2017. Wireless power transfer inspired by the modern trends in electromagnetics. Applied Physics Reviews 4, 2 (March 2017), 021102. https://doi.org/10.1063/1.4981396

[40] Vamsi Talla, Mehrdad Hessar, Bryce Kellogg, Ali Najafi, Joshua R. Smith, and Shyamnath Gollakota. 2017. LoRa Backscatter: Enabling The Vision of Ubiquitous Connectivity. Proceedings of the ACM on Interactive, Mobile, Wearable and Ubiquitous Technologies (IMWUT) 1 , 3, Article 105 (Sept. 2017), 24 pages. https://doi.org/10.1145/3130970

[41] Vamsi Talla, Bryce Kellogg, Shyamnath Gollakota, and Joshua R. Smith. 2017. Battery-Free Cellphone. Proceedings of the ACM on Interactive, Mobile, Wearable and Ubiquitous Technologies (IMWUT) 1, 2, Article 25 (June 2017), 20 pages. https://doi.org/10.1145/3090090

[42] Keysight Technologies. [n. d.]. Advanced Design System (ADS). ([n. d.]). "https://www.keysight.com/en/pc-1297113/ advanced-design-system-ads (Accessed on May 15, 2018)".

[43] Ryan Tseng, Bill von Novak, Sumukh Shevde, and Kamil A Grajski. 2013. Introduction to the alliance for wireless power loosely-coupled wireless power transfer system specification version 1.0. In Proceedings of the 2013 IEEE Wireless Power Transfer (WPT). IEEE, 79-83. https://doi.org/10.1109/WPT.2013.6556887

[44] Dries Van Wageningen and Toine Staring. 2010. The Qi wireless power standard. In Proceedings of the 2010 14th International Power Electronics and Motion Control Conference (EPE/PEMC). IEEE, S15-25. https://doi.org/10.1109/EPEPEMC.2010.5606673

[45] Mark Weiser. 1991. The Computer for the 21 st Century. Scientific american 265, 3 (1991), 94-105.

[46] Haifeng Wu, Yu Zeng, Jihua Feng, and Yu Gu. 2013. Binary tree slotted ALOHA for passive RFID tag anticollision. IEEE Transactions on Parallel and Distributed Systems 24, 1 (April 2013), 19-31. https://doi.org/10.1109/TPDS.2012.120

[47] Chouchang (Jack) Yang, Jeremy Gummeson, and Alanson P Sample. 2017. Riding the airways: Ultra-wideband ambient backscatter via commercial broadcast systems. In Proceedings of the IEEE INFOCOM 2017 - IEEE Conference on Computer Communications. 1-9. https://doi.org/10.1109/INFOCOM.2017.8057162

[48] Meysam Zargham and P Glenn Gulak. 2012. Maximum achievable efficiency in near-field coupled power-transfer systems. IEEE Transactions on Biomedical Circuits and Systems 6, 3 (January 2012), 228-245. https://doi.org/10.1109/TBCAS.2011.2174794

[49] Yong Zeng, Bruno Clerckx, and Rui Zhang. 2017. Communications and Signals Design for Wireless Power Transmission. IEEE Transactions on Communications 65, 5 (May 2017), 2264-2290. https://doi.org/10.1109/TCOMM.2017.2676103

[50] Yujuan Zhao, Lin Tang, Robert Rennaker, Chris Hutchens, and Tamer S. Ibrahim. 2013. Studies in RF Power Communication, SAR, and Temperature Elevation in Wireless Implantable Neural Interfaces. PLOS ONE 8, 11 (November 2013). https://doi.org/10.1371/journal. pone.0077759

Received May 2018; revised August 2018; accepted October 2018

Proc. ACM Interact. Mob. Wearable Ubiquitous Technol., Vol. 2, No. 4, Article 188. Publication date: December 2018. 